\title{
Myocardial infarction detection using artificial intelligence and nonlinear features based on synthesis of the standard 12-lead and Frank XYZ leads
}

\author{
Wei Zeng ( $\nabla$ zengwei@lyun.edu.cn ) \\ Longyan University https://orcid.org/0000-0002-8353-8265 \\ Zixiang Lin \\ Longyan University \\ Chengzhi Yuan \\ University of Rhode Island
}

\section{Research}

Keywords: myocardial infarction (MI) detection, electrocardiographic (ECG), intrinsic time-scale decomposition (ITD), discrete wavelet transform (DWT), cardiac system dynamics, neural networks

Posted Date: August 13th, 2020

DOI: https://doi.org/10.21203/rs.3.rs-56003/v1

License: (c) (i) This work is licensed under a Creative Commons Attribution 4.0 International License.

Read Full License 


\title{
Myocardial infarction detection using artificial intelligence and nonlinear features based on synthesis of the standard 12-lead and Frank XYZ leads
}

\author{
Wei Zeng ${ }^{1 *}$, Zixiang $\operatorname{Lin}^{1}$ and Chengzhi Yuan ${ }^{2}$
}

\author{
${ }^{*}$ Correspondence: \\ zengwei@lyun.edu.cn \\ ${ }^{1}$ School of Physics and \\ Mechanical and Electrical \\ Engineering, Longyan University, \\ Longyan 364012, China \\ Full list of author information is \\ available at the end of the article
}

\begin{abstract}
Nowadays cardiovascular diseases (CVD) is one of the prime causes of human mortality, which has received tremendous and elaborative research interests regarding the prevention of CVD. Myocardial ischemia is a kind of CVD which will lead to myocardial infarction (MI). The diagnostic criterion of $\mathrm{MI}$ is supplemented with clinical judgment and several electrocardiographic (ECG) or vectorcardiographic (VCG) programs. However the visual inspection of ECG or VCG signals by cardiologists is tedious, laborious and subjective. To overcome such disadvantages, numerous MI detection techniques including signal processing and artificial intelligence tools have been developed. In this study we propose a novel technique for automatic detection of MI based on disparity of cardiac system dynamics and synthesis of the standard 12-lead and Frank XYZ leads. First, 12-lead ECG signals are reduced to 3-dimensional VCG signals, which are synthesized with Frank XYZ leads to build a hybrid 4-dimensional cardiac vector. This vector is decomposed into a series of proper rotation components (PRCs) by using the intrinsic time-scale decomposition (ITD) method. Second, four levels discrete wavelet transform (DWT) is employed to decompose the predominant PRCs into different frequency bands, in which third-order Daubechies (db3) wavelet function is selected as reference variable for analysis. Third, phase space of the reference variable is reconstructed based on $\mathrm{db} 3$, in which the properties associated with the nonlinear cardiac system dynamics are preserved. Three-dimensional (3D) phase space reconstruction (PSR) together with Euclidean distance (ED) has been utilized to derive features. Fourth, neural networks are then used to model, identify and classify cardiac system dynamics between normal (healthy) and MI cardiac vector signals. Finally, experiments are carried out on the PhysioNet PTB database to assess the effectiveness of the proposed method, in which conventional 12-lead and Frank XYZ leads ECG signal fragments from 148 patients with $\mathrm{MI}$ and 52 healthy controls were extracted. By using the 10 -fold cross-validation style, the achieved average classification accuracy is reported to be $98.20 \%$. The result verifies the effectiveness of the proposed method which can serve as a potential candidate for the automatic detection of $\mathrm{MI}$ in the clinical application.
\end{abstract}

Keywords: myocardial infarction (MI) detection; electrocardiographic (ECG); intrinsic time-scale decomposition (ITD); discrete wavelet transform (DWT); cardiac system dynamics; neural networks 


\section{Introduction}

Cardiovascular diseases (CVD) are the most common cause of human mortality [1]. Measurement of timing intervals and amplitudes of the electrocardiographic (ECG) or vectorcardiographic (VCG) waves provides fundamental feature in CVD diagnostics. Myocardial ischemia is a kind of CVD which will lead to myocardial infarction (MI). Pathological alterations provoked by MI cause slow conduction by increasing axial resistance on coupling between cells. This issue may cause abnormal patterns in the dynamics of the tip of the cardiac vector [2]. Hence analysis of the morphology of ECG or VCG recordings plays an important role in monitoring the variation of dynamics in the pathological alterations of cardiac patterns caused by MI. In order to diagnose CVD, the standard 12-lead ECG has been regarded as the gold standard for monitoring and analyzing alternations in the cardiac activity [3]. From the perspective of medical science, cardiologists have been accustomed to the standard 12-lead system due to its noninvasiveness, inexpensiveness and availability for CVD diagnosis. Traditionally, detection of MI based on the visual inspection of ECG or VCG signals by cardiologists is tedious, laborious and subjective [4]. In addition, it requires expertise in the analysis of long-term ECG or VCG signals [5]. In those application scenarios absence of experts, for example, in emergency situations, computer-aided automatic detection of MI becomes significant.

To overcome the aforementioned disadvantages of the standard 12-lead ECG system, strategies can be taken in two aspects: (1) reduce the number of leads; and (2) employ artificial intelligence tools to analyze and classify cardiac patterns. In the first aspect, several studies have proposed to reduce the number of leads that can reconstruct the 12-lead ECG signals without loss of significant information in diagnosis. These methods can be categorized into two types: using subsets of the 12-lead and using special leads [4, 6]. For the first category, subsets of the leads of the 12-lead ECG are used to reconstruct the standard 12-lead ECG [7-12]. The missing precordial leads are reconstructed from the remaining leads by using either a personalized or a universal transformation. For the second category, special leads are used based on the orthogonal lead system. For example, the orthogonal Frank XYZ leads and EASI leads are well-known for use of fewer leads compared to the widely used 12-lead ECG systems, since it is convenient to implement and can capture more information than Holter leads [6, 13-16].

In the second aspect, ECG or VCG signal based arrhythmia or MI detection techniques including signal processing and artificial intelligence tools have been developed, such as linear [17, 18] and nonlinear methods [19, 20], Wavelet Transform (WT) [21], Complex Wavelet Transform (CWT) [22, 23], Pitch Synchronous Wavelet Transform (PSWT) [24], Discrete Wavelet Transform (DWT) [25], Kalman filtering (KF) [26], Least Mean Squares algorithm (LMS) [27], ensemble learning [28, 29], Artificial Neural Networks (ANN) [30], Adaptive Neuro-fuzzy Inference System (ANFIS) [31], support vector machine (SVM) [20], and deep learning [3242]. For example, Varatharajan et al. [43] used linear discriminant analysis (LDA) to reduce the features presented in the ECG signal, which followed with a SVM model with a weighted kernel function for the classification of cardiac arrhythmia. Plawiak [44] used the spectral power density to enhance feature performance extracted from ECG signals and employed genetic algorithms for feature selection. 
Then several machine learning tools including SVM, k-nearest neighbor, ANN, have been used for the classification of cardiac disorders. Kumar and Inbarani [29] used DWT to extract effective feautures. Then neighborhood rough set was employed for the ECG signal classification. Acharya et al. [35] used ECG signals of two seconds and five seconds' durations as the input of convolutional neural network (CNN) with eleven-layer to detect different types of cardiac arrhythmia. There is no need for the pre-processing of QRS detection. Kumar et al. [45] used flexible analytic wavelet transform (FAWT) to decompose ECG beats into subband signals. Then sample entropy was computed as features and fet into random forest (RF), J48 decision tree, back propagation neural network (BPNN), and least-squares support vector machine (LS-SVM) classifiers for detection of MI. Yildirim [37] decomposed ECG signals into frequency sub-bands at different scales, which were used as the input of long-short term memory networks (LSTMs) for ECG signal classification. However, it still remains an open problem for the automatic arrhythmia or MI detection with high efficiency and accuracy based on different types of lead system in discriminating between normal and abnormal ECG signals [46].

Because of the redundant and discrete-time characteristics of ECG and VCG signals, numerous methods with combination of time and frequency domains and nonlinear analysis have been developed to handle the problem [47]. Especially for the nonlinear analysis, nonlinear parameters extracted through different types of entropies [48], Lyapunov exponent [49], local fractal dimension [50], higher order spectra (HOS) cumulants [51], recurrence quantification analysis (RQA) [52] and Hurst exponent [53], have been employed for automatic detection of abnormal ECG or VCG signals. Despite the fact that the above-mentioned methods have achieved respectable classification accuracy, the potential of nonlinear methods, including the nonlinear features and the modeling and identification algorithms for nonlinear cardiac system dynamics, has not been thoroughly investigated. Considering the characteristics that the ECG signal is highly random, nonlinear, nonstationary and non-Gaussian in nature [54], self-adaptive signal processing methods, such as empirical mode decomposition (EMD) [55, 56], local mean decomposition (LMD) [57] and intrinsic time-scale decomposition (ITD) [58], can be employed to extract effective and predominant features from ECG or VCG signals [59, 60]. In comparison to EMD and LMD, ITD significantly improves the computational efficiency. In addition, with high decomposition efficiency and frequency resolution, ITD can help decompose a complex signal into several proper rotation components (PRCs) and a baseline signal, which leads to the accurate extraction of the dynamic features of nonlinear signals. In the present work we have developed a novel algorithm to compute the representative features based on ITD algorithm and the synthesis of 12-lead and Frank XYZ leads. We hypothesize that these features reflect the abnormal alterations in the dynamics of the cardiac vector and can achieve high sensitivity and specificity simultaneously as a discriminator of MI.

The novelty of this study lies in four aspects: 1) we use the synthesis of 12-lead and Frank XYZ leads to build a new 4-dimensional cardiac vector which may fully reflect the pathological alterations provoked by MI. 2) ITD method is employed to measure the variability of cardiac vector and the first proper rotation components (PRCs) are extracted as predominant PRCs which contain most of the cardiac 
vector's energy. 3) Discrete wavelet transform (DWT) decomposes the predominant PRCs into different frequency bands, which are used to construct the reference variables. 4) 3D phase space of the predominant PRCs components is reconstructed, in which the properties associated with the cardiac system dynamics are preserved. 5) Cardiac system dynamics can be modeled and identified using neural networks, which employ the ED of 3D PSR of the reference variables as input features. 6) The difference of cardiac system dynamics between healthy control and MI cardiac vector is computed and used for the detection of MI based on a bank of estimators.

\section{Method}

In this section, we propose a method to discriminate between normal and MI cardiac vectors using the information obtained from nonlinear cardiac system dynamics for the detection of MI. It is divided into the training stage and the classification stage, which includes the following steps. In the first step, the synthesis of 12-lead and Frank XYZ leads is used to build a new 4-dimensional cardiac vector. In the second step, ITD is applied to decompose the cardiac vector into several PRCs to extract $\mathrm{PRC}_{1}$ predominant components. In the third step, DWT is employed to decompose the predominant PRCs into different frequency bands. In the fourth step, PSR is applied to extract nonlinear dynamics of cardiac system and Euclidean distances are computed. Finally, feature vectors are fed into the neural networks for the modeling and identification of cardiac system dynamics. The difference of dynamics between normal (healthy) and MI cardiac vector will be applied for the classification task. The flowchart of the proposed algorithm is illustrated in Fig. 1.

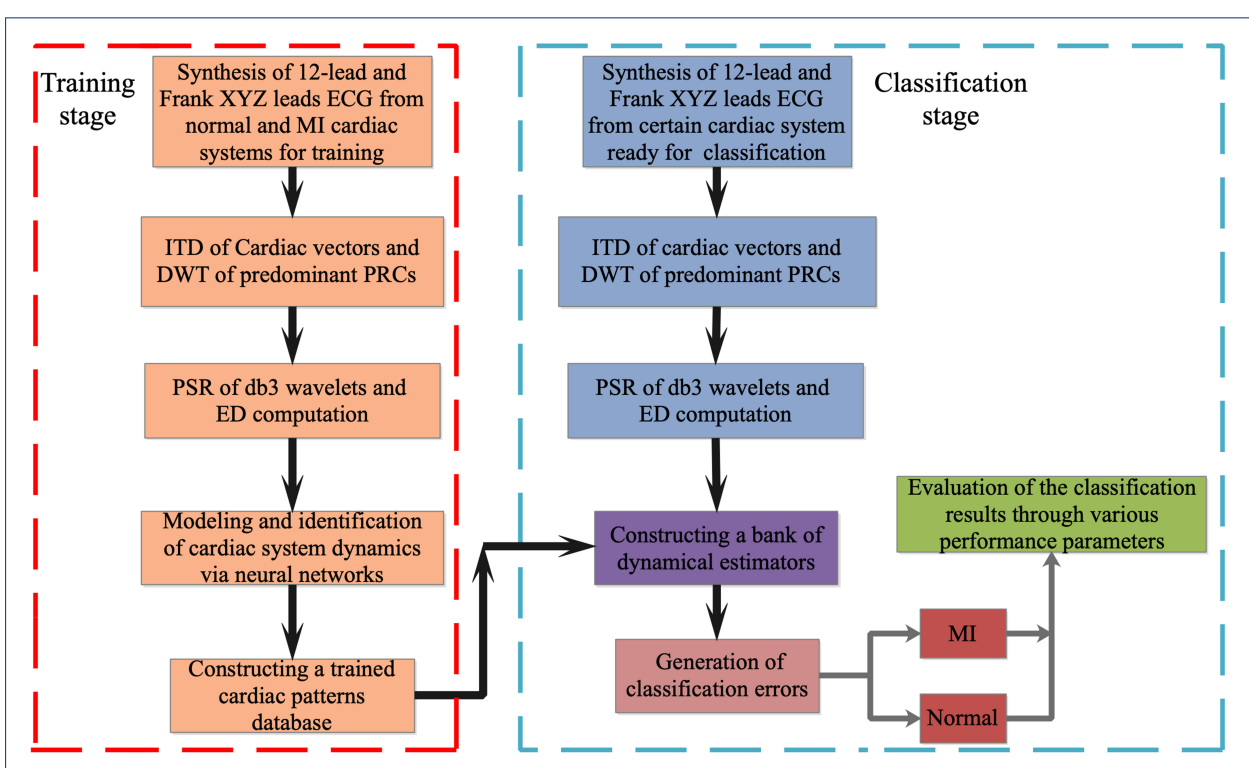

Fig. 1 Flowchart of the proposed method for the MI classification using syntheses of lead system, ITD, DWT, PSR, ED and neural networks.

\subsection{ECG database}

In this study we utilize the popular and publicly available PTB database which has been acquired at the Department of Cardiology of University Clinic Benjamin 
Franklin in Berlin, Germany, and has been provided to the users of PhysioNet $[61,62]$. The database contains 549 records from 290 subjects, in which there are 148 patients with MI and 52 healthy control (HC) subjects. Each subject is represented by one to five records. Hence there are 367 and 78 heart beat records for MI and HC subjects, respectively. Each record includes 15 simultaneously measured signals: the conventional 12 leads (i, ii, iii, avr, avl, avf, v1, v2, v3, v4, v5, v6) together with the 3 Frank XYZ leads ECGs (vX, vY, vZ). Each signal was digitized at 1000 samples per second.

In this study, the characteristics of the used ECG data obtained from PTB database is presented in Table 1.

Table 1 Description of the PTB database with the selected ECG signals.

\begin{tabular}{lll}
\hline Characteristics & HC & MI \\
\hline Age range & $17-81$ & $36-86$ \\
Average age & 43.4 & 60.4 \\
Number of participants & 52 & 148 \\
Number of heart beat records & 78 & 367 \\
Ratio of male to female & 3 & 2.89 \\
\hline
\end{tabular}

2.2 Synthesis of 12-lead and Frank XYZ leads

First, transform the 12-lead ECG into 3-dimensional VCG since VCG signals and 12-lead ECG signals can be linearly transformed into each other without loss of useful information content pertaining to the cardiac system dynamics [63]. The transformation relationship is shown as follows [64]:

$$
\left\{\begin{array}{l}
V_{1}=0.38 x_{1}-0.07 x_{2}-0.13 x_{7}+0.05 x_{8}-0.01 x_{9}+0.14 x_{10}+0.06 x_{11}+0.54 x_{12} \\
V_{2}=-0.07 x_{1}+0.93 x_{2}+0.06 x_{7}-0.02 x_{8}-0.05 x_{9}+0.06 x_{10}-0.17 x_{11}+0.13 x_{12} \\
V_{3}=0.11 x_{1}-0.23 x_{2}-0.43 x_{7}-0.06 x_{8}-0.14 x_{9}-0.20 x_{10}-0.11 x_{11}+0.31 x_{12}
\end{array}\right.
$$

where $x_{1}$ to $x_{12}$ represent the 12 leads (i, ii, iii, avr, avl, avf, v1, v2, v3, v4, v5, v6), respectively, $V_{1}, V_{2}, V_{3}$ represent the transformed $3 \mathrm{VCG}$ signals, respectively. For the Frank XYZ leads,

$$
V_{4}=\sqrt{X^{2}+Y^{2}+Z^{2}}
$$

where $X, Y$ and $Z$ represent the lead value of three corresponding axes, $V_{4}$ represents the VCG voltage magnitude of the 3 Frank leads by taking the square root of the sum of the squares for the three individual axes.

Here we build a new 4-dimensional cardiac vector: $V=\left[V_{1}, V_{2}, V_{3}, V_{4}\right]^{T}$, which contains useful information content from the 12-lead and Frank XYZ leads. It reduces the dimension of feature vectors instead of using 15 leads ECG signals directly. In addition, the novel cardiac vector may fully reflect the pathological alterations provoked by MI and may be correlated to the disparity of cardiac system dynamics between $\mathrm{HC}$ and MI subjects. The examples of the 4-dimensional cardiac vectors from $\mathrm{HC}$ and $\mathrm{MI}$ subjects are demonstrated in Figs. 2 and 3. 


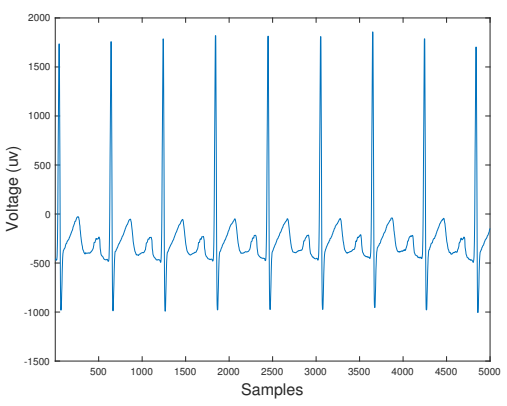

(a) $V_{1}$

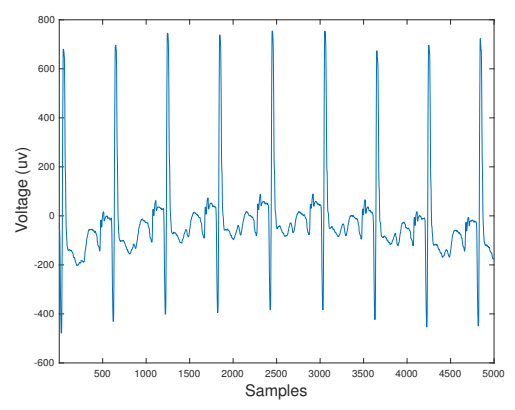

(c) $V_{3}$

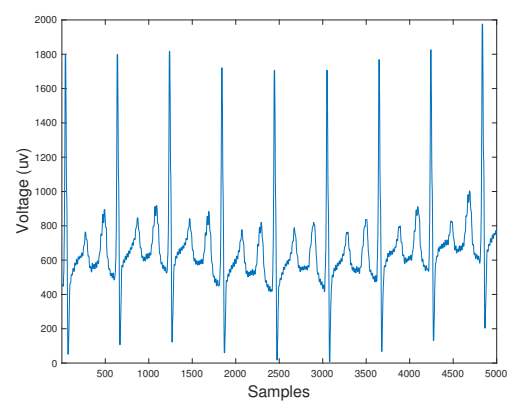

(b) $V_{2}$

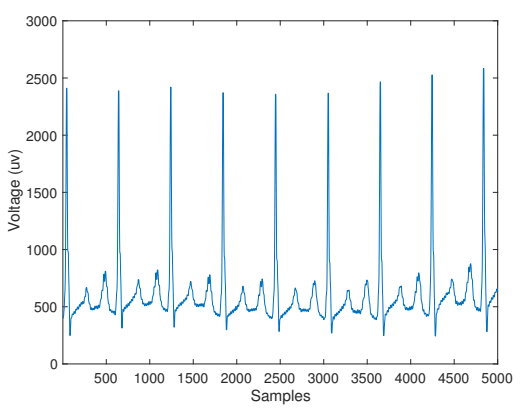

(d) $V_{4}$

Fig. 2 Examples of the synthesis of 4-dimensional cardiac vector: $V=\left[V_{1}, V_{2}, V_{3}, V_{4}\right]^{T}$ from $\mathrm{HC}$ subject. 


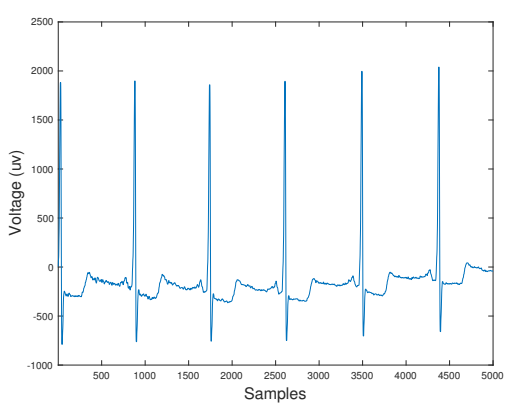

(a) $V_{1}$

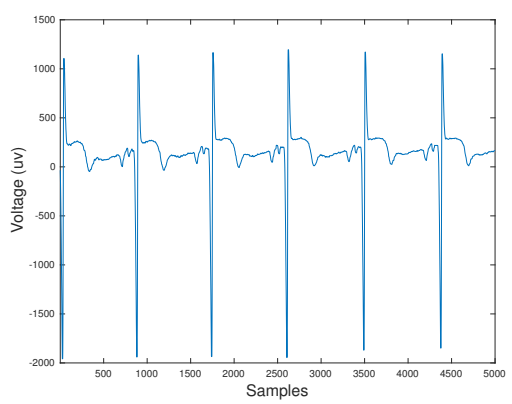

(c) $V_{3}$

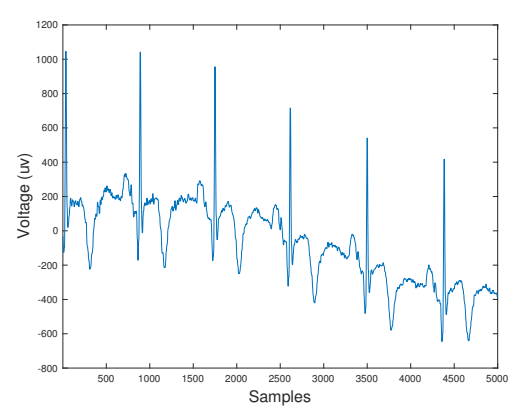

(b) $V_{2}$

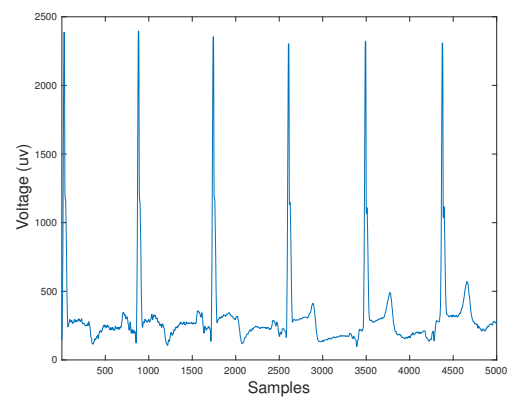

(d) $V_{4}$

Fig. 3 Examples of the synthesis of 4-dimensional cardiac vector: $V=\left[V_{1}, V_{2}, V_{3}, V_{4}\right]^{T}$ from MI subject. 


\subsection{Intrinsic time-scale decomposition (ITD)}

Intrinsic time-scale decomposition (ITD) is suitable for analyzing nonstationary and nonlinear signals such as the ECG and VCG signals. Without resorting to the spline interpolation to signal extrema and sifting in mono-component separation, it decomposes a signal into proper rotation components (PRCs) that are suitable to calculate the instantaneous frequency and amplitude, based on the baseline defined via linear transform. The obtained decomposition result precisely preserves the temporal information of each component regarding signal critical points and riding waves, with a time resolution equal to the time scale of the occurrence of extrema in the raw signal [65]. Based on the single wave analysis, it extracts accurately the inherent instantaneous amplitude and frequency/phase information and other relevant morphological features [58].

For a time series signal $I(t)$, define the operator $L$ to extract the baseline signal from $I(t)$ and the residual signal is called the proper rotation component (PRC). The decomposed signal $I(t)$ can be expressed as

$$
I(t)=L I(t)+(1-L) I(t)=B(t)+H(t)
$$

where $B(t)$ is the baseline signal and $H(t)$ is the proper rotation.

The decomposition procedure of a nonlinear signal can be summarized by the following steps:

- Step 1: Find the local extrema of the signal $I(t)$, denoted by $I_{k}$, and the corresponding occurrence time instant $\tau_{k}, k=0,1,2, \cdots$. For convenience $\tau_{0}=$ 0 .

- Step 2: Suppose the operators $B(t)$ and $H(t)$ are given over the interval $\left[0, \tau_{k}\right]$, and $I(t)$ is set on the interval $t \in\left[0, \tau_{k+2}\right]$. Then on the interval $\left[\tau_{k}, \tau_{k+1}\right]$ between two adjacent extrema $I_{k}$ and $I_{k+1}$, the piecewise baseline extraction operator is defined as

$$
L I(t)=B(t)=B_{k}+\left(\frac{B_{k+1}-B_{k}}{I_{k+1}-I_{k}}\right) \times\left(I(t)-I_{k}\right), t \in\left[\tau_{k}, \tau_{k+1}\right],
$$

where

$$
B_{k+1}=\beta\left[I_{k}+\left(\frac{\tau_{k+1}-\tau_{k}}{\tau_{k+2}-\tau_{k}}\right)\left(I_{k+2}-I_{k}\right)\right]+(1-\beta) I_{k+1},
$$

and $0<\beta<1$, typically $\beta=0.5$.

- Step 3: After extracting the baseline signal, the operator $\Theta$ for extracting the residual signal as PRCs is defined as

$$
\Theta I(t) \equiv(1-L) I(t)=I(t)-B(t)
$$

According to the definition, the PRC is a riding wave with the highest frequency on the baseline. Therefore, ITD separates the PRC in a frequency order from high to low. In addition, the PRC is obtained directly by subtracting the baseline from the input signal, without resorting to any sifting within each iterative decomposition. 
Thus, ITD has low computational complexity, and more importantly, avoids the smoothing of transients and time-scale smearing due to repetitive sifting [65].

Take the baseline $B(t)$ as the input signal $I(t)$, and repeat steps (1)-(3), until the baseline becomes a monotonic function or a constant. Eventually, the raw signal will be decomposed into PRCs and a trend [65]

$$
I(t)=\sum_{i=1}^{\rho} H^{i}(t)+B^{\rho}(t)
$$

where $\rho$ is the decomposition level.

Samples of the ITD of 4-dimensional cardiac vector from the HC and MI subjects are demonstrated in Figs. 4 and 5.
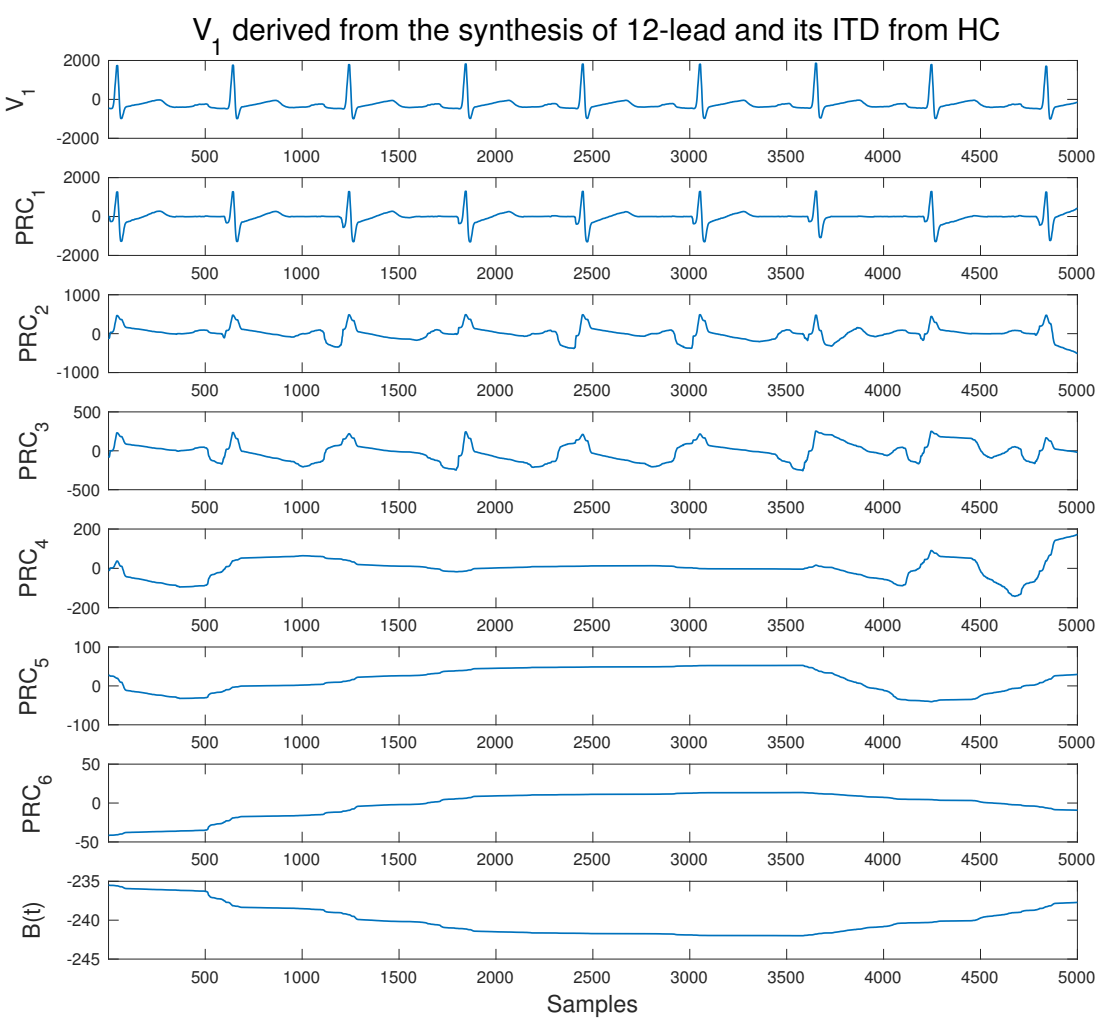

(a) Original $V_{1}$ vector and its ITD from HC. 

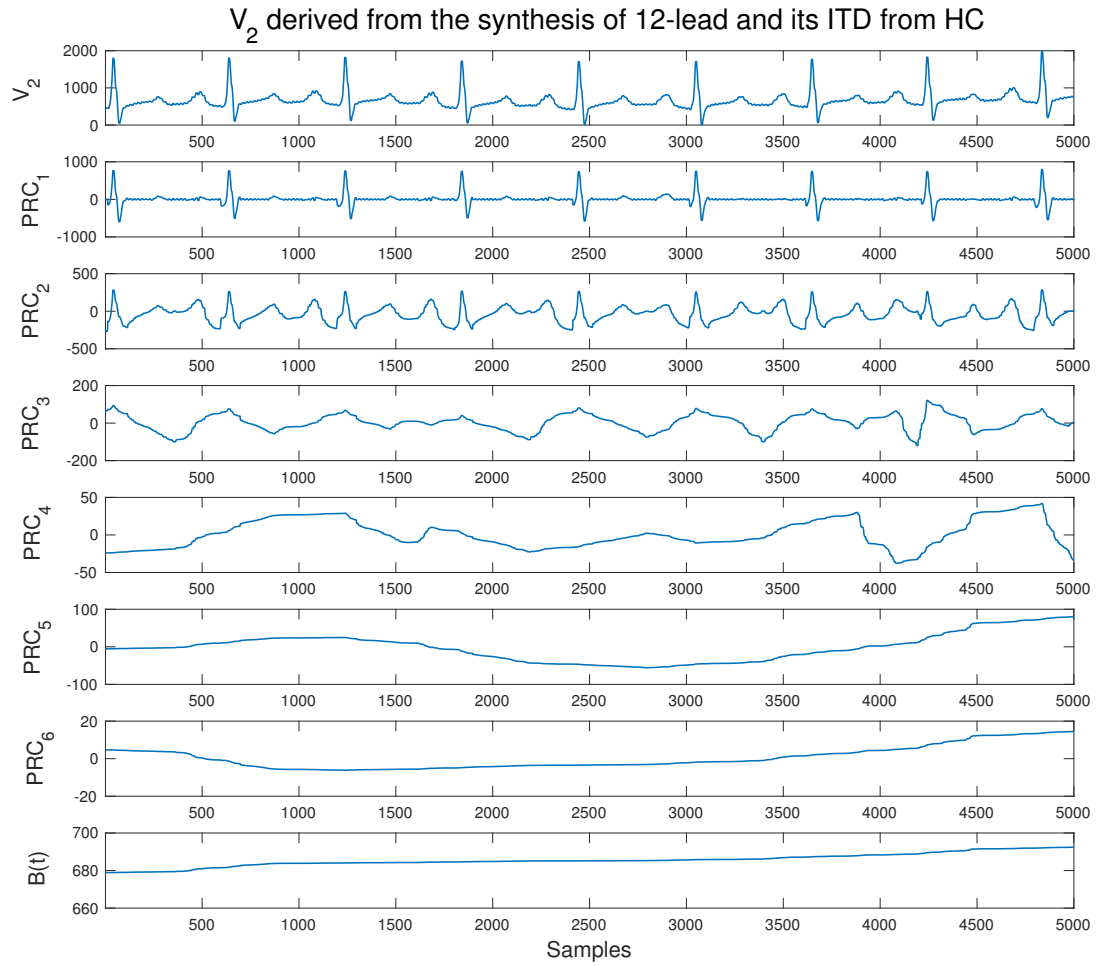

(b) Original $V_{2}$ vector and its ITD from $\mathrm{HC}$ 

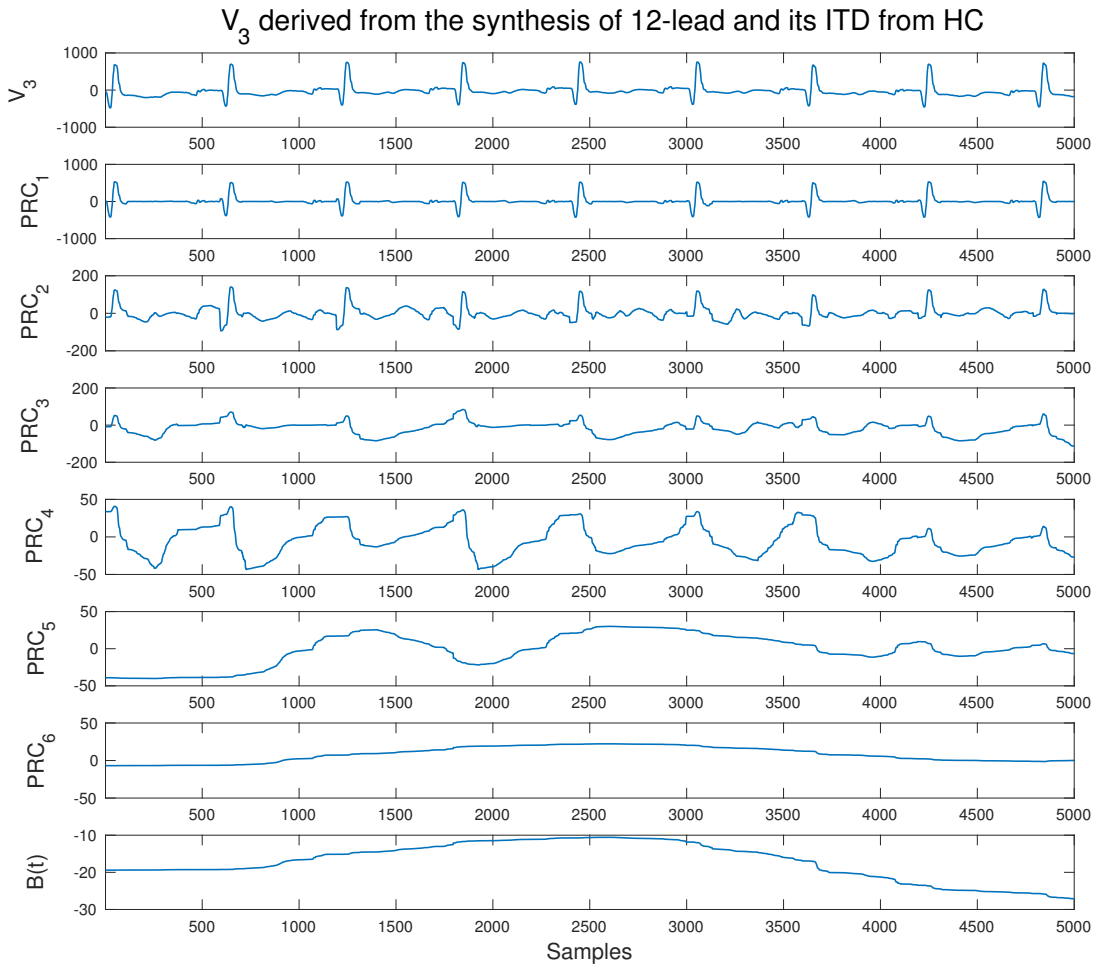

(c) Original $V_{3}$ vector and its ITD from HC 

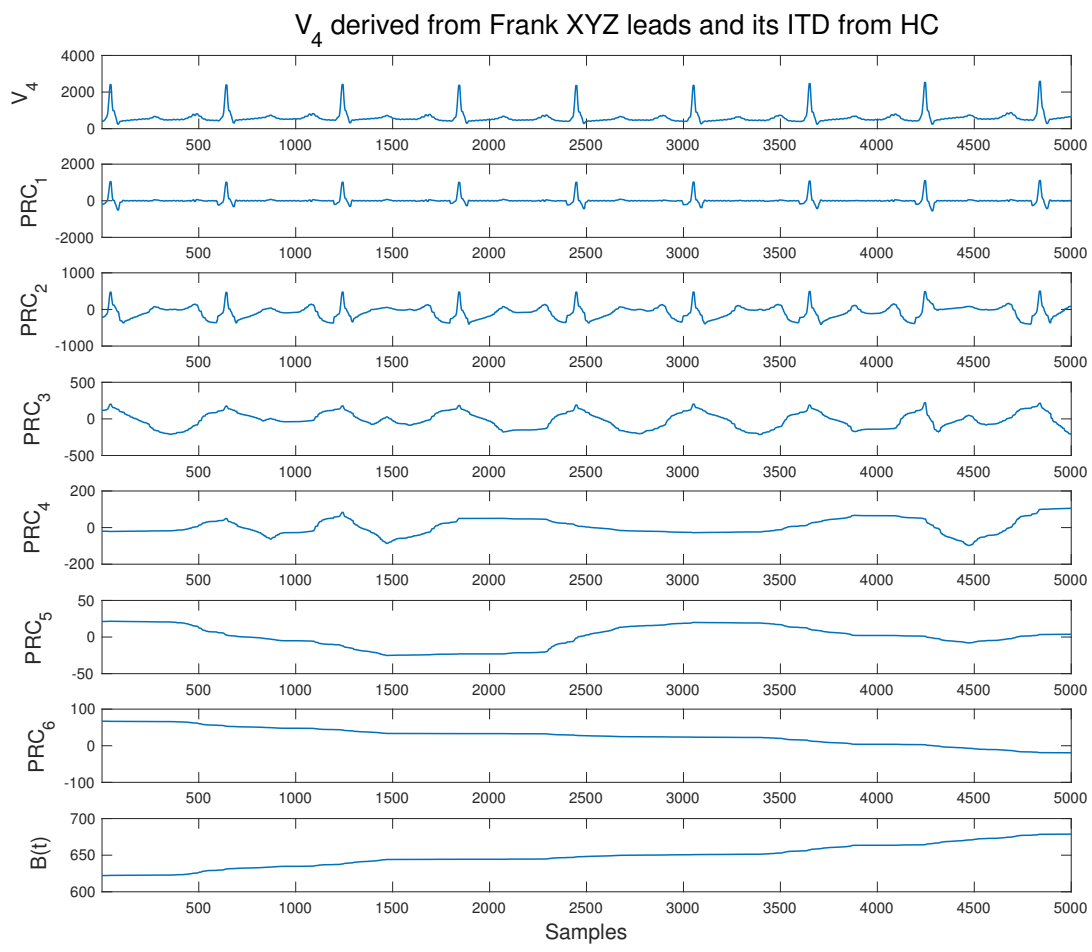

(d) Original $V_{4}$ vector and its ITD from $\mathrm{HC}$

Fig. 4 Samples of 4-dimensional cardiac vector: $V=\left[V_{1}, V_{2}, V_{3}, V_{4}\right]^{T}$ from $\mathrm{HC}$ subject. 

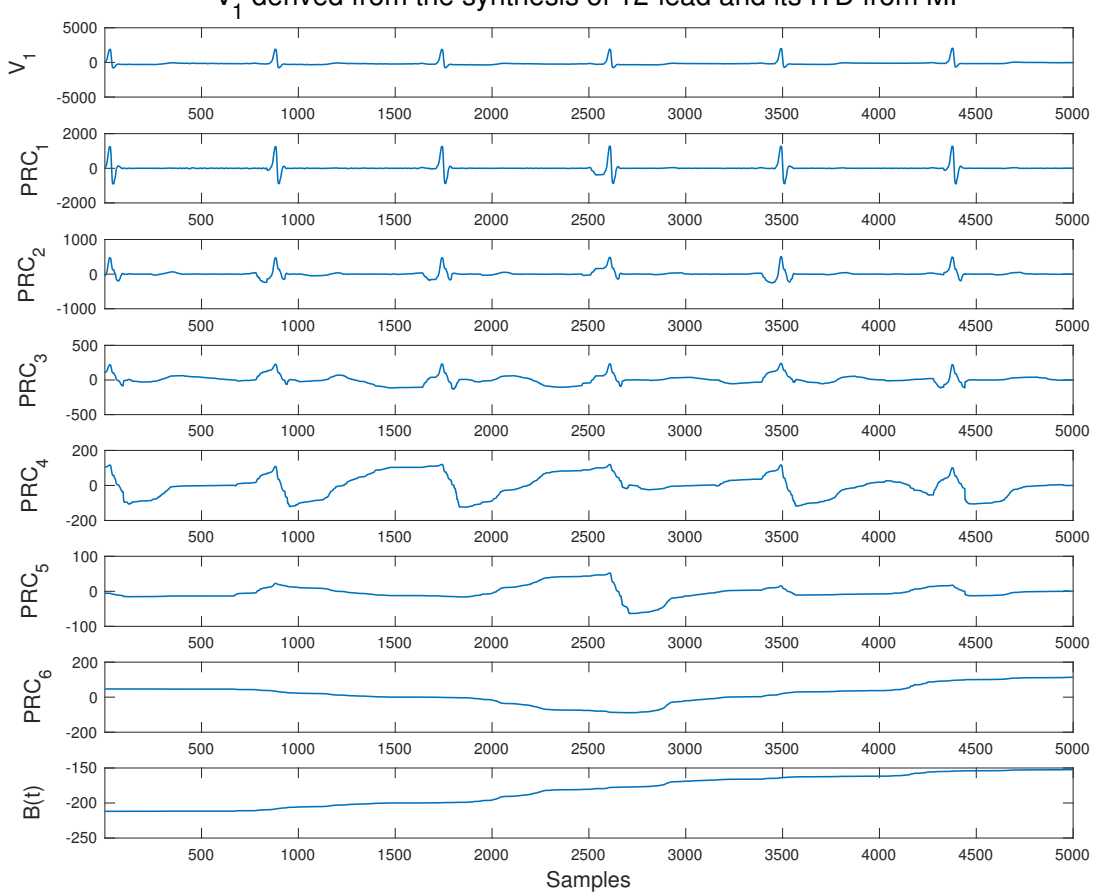

(a) Original $V_{1}$ vector and its ITD from MI. 

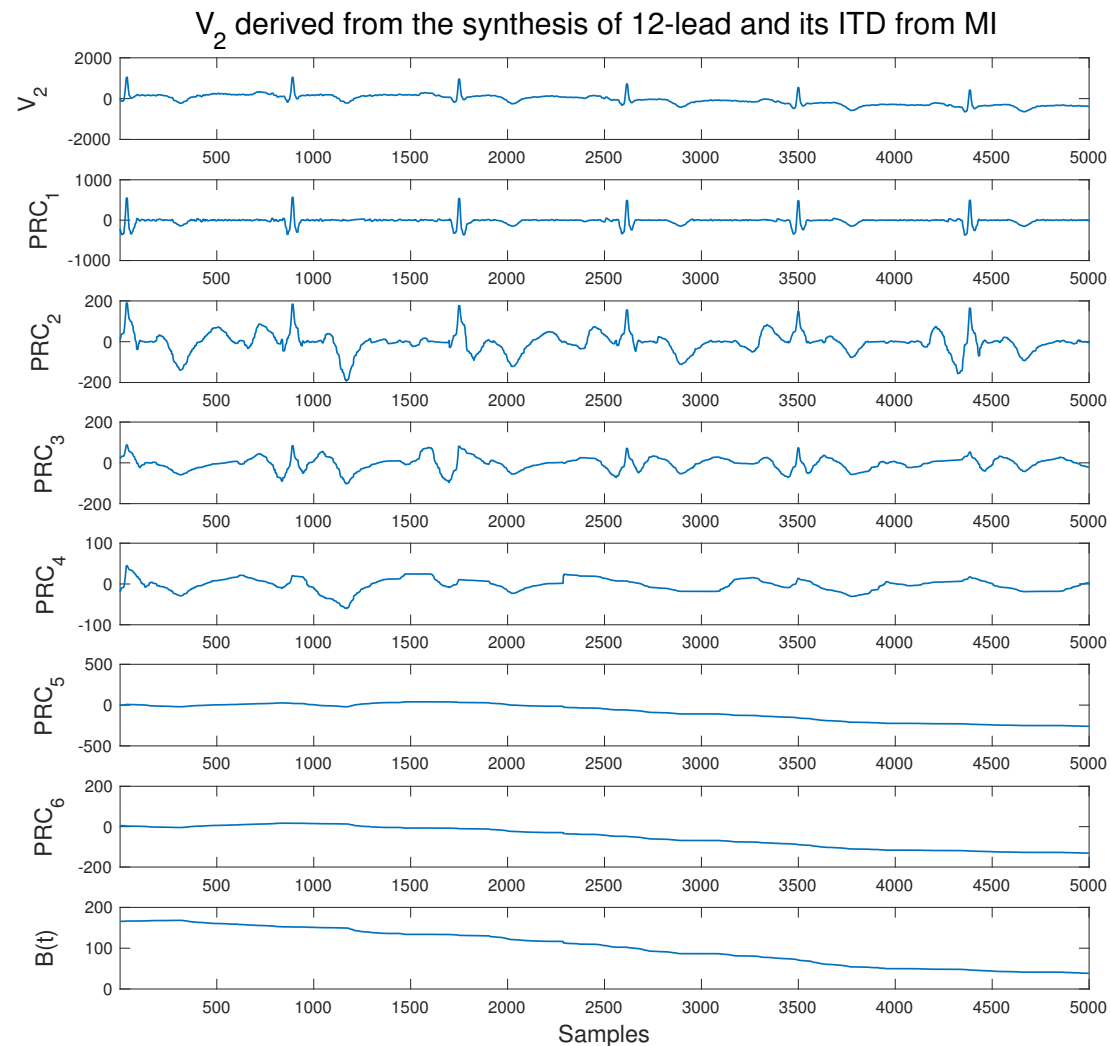

(b) Original $V_{2}$ vector and its ITD from MI 


\subsection{Discrete wavelet transform (DWT)}

Wavelet transform is an effective time-frequency tool for the analysis of nonstationary signals. Discrete Wavelet Transform (DWT) is a procedure for the decomposition of input signal $H(t)(H(t)$ is the PRC of the cardiac vector in this work) into sets of function, called wavelets, by scaling and shifting of mother wavelet function. Consequently, the decomposition i.e. set of wavelet coefficients are formed.

To accomplish this, the signal $H(t)$ can be reconstructed as linear combination of wavelets and weighting wavelet coefficients. The setting of appropriate wavelet function and the number of decomposition levels is of great importance for correctly reconstructing the signal $H(t)$. It was reported that Most of the energy of the ECG signal lies between $0.5 \mathrm{~Hz}$ and $40 \mathrm{~Hz}$ [66], for which the wavelet coefficients is concentrated in the lower sub-bands. In order to extract five physiological cardiac vector bands, four levels DWT with third-order Daubechies (db3) wavelet function have been used (Table 2 represents the frequency distribution of the DWT-based coefficients of the PRCs of the cardiac vector at $1000 \mathrm{~Hz}$ ), from which the choice of the mother wavelet is supported by many works in literature [67-69]. Fig. 6 shows samples of cardiac vector channel of $\mathrm{HC}$ and MI subjects and their decomposed frequency bands of predominant PRCs. Since the frequency components above 40 $\mathrm{Hz}$ is lack of use in ECG analysis, in order to reduce the feature dimension, the advisable sub-band (D4) is selected for feature acquisition. The detailed information of levels 1, 2 and 3 (sub-bands D1, D2 and D3) are discarded since the frequencies covered by these levels are higher than frequency content of the cardiac vector.

Table 2 Frequency band of PRCs of the cardiac vector using fourth level decomposition of DWT

\begin{tabular}{lll}
\hline Decomposition levels & Sub-bands & Frequency range $(\mathrm{Hz})$ \\
\hline 1 & D1 & $250-500$ \\
2 & D2 & $125-250$ \\
3 & D3 & $62.5-125$ \\
4 & D4 & $31.25-62.5$ \\
4 & A4 & $0-11.25$ \\
\hline
\end{tabular}


1
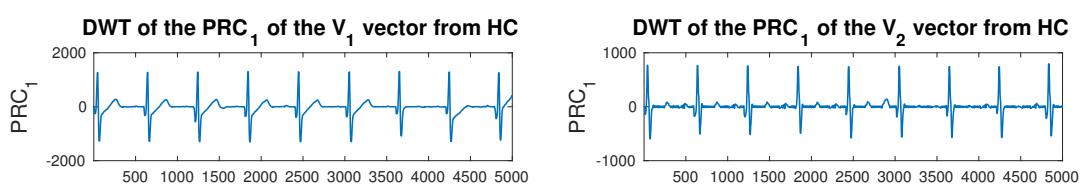

$\overline{0}$
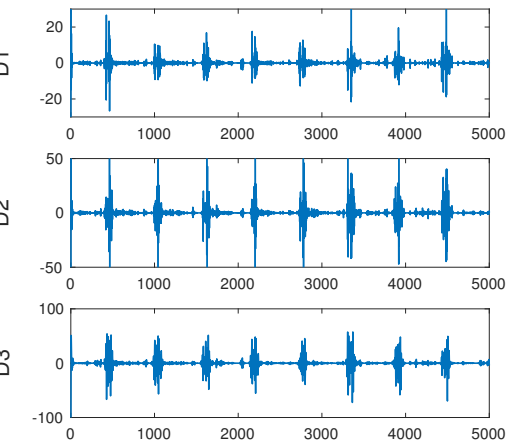

\&
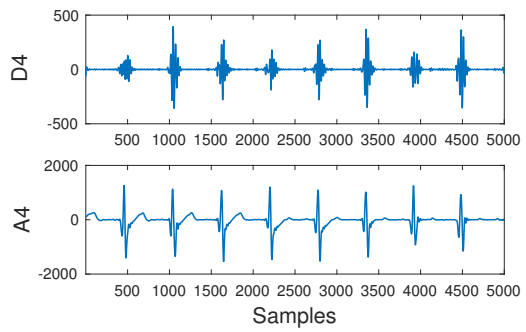
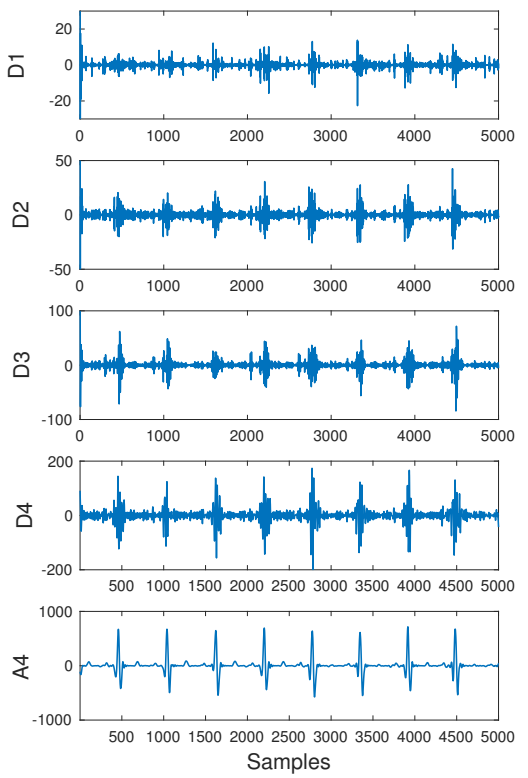

(a) Four levels DWT of $\mathrm{PRC}_{1}$ of the $V_{1}$ and $V_{2}$ vectors from $\mathrm{HC}$. 

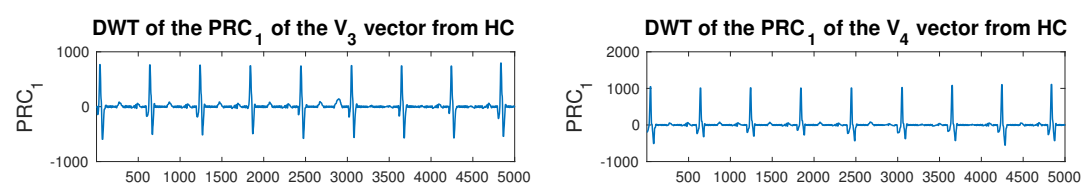

$\bar{\square}$

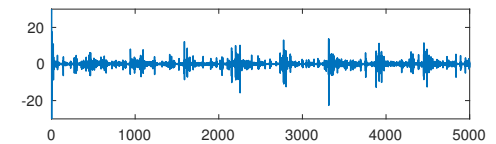

ธิ
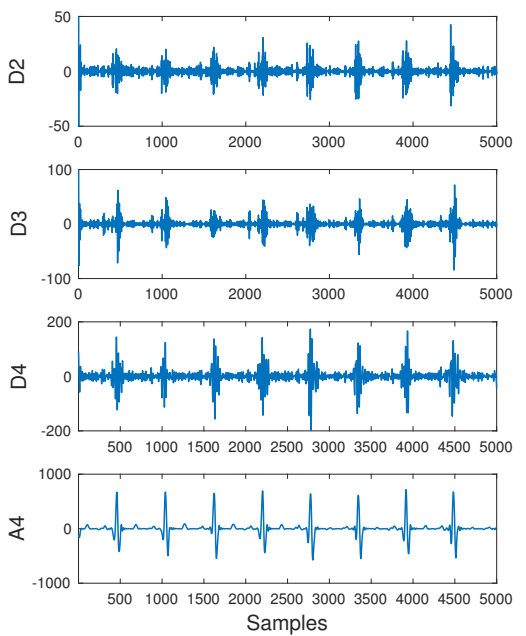

$\bar{\square}$

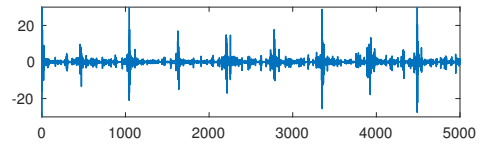

ธิ

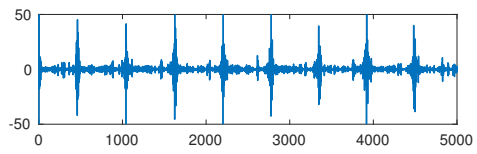

ติ

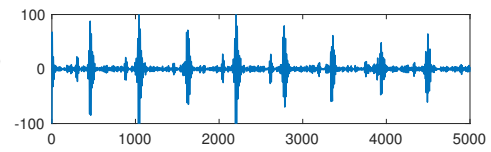

范
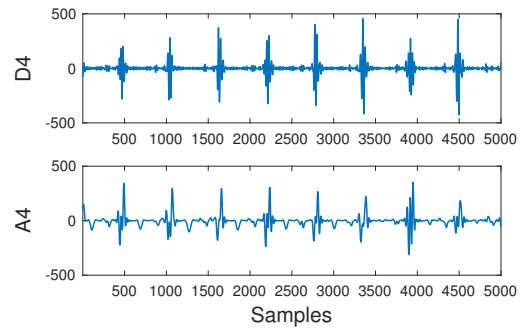

(b) Four levels DWT of $\mathrm{PRC}_{1}$ of the $V_{3}$ and $V_{4}$ vectors from $\mathrm{HC}$. 

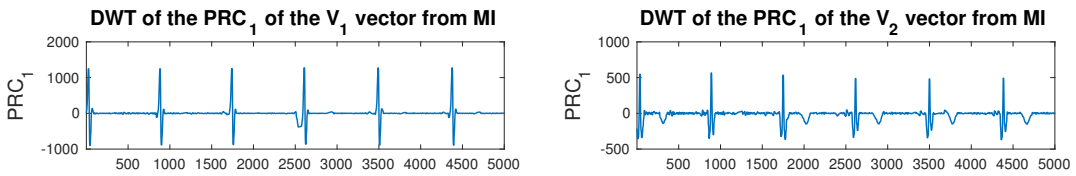

$\bar{\square}$

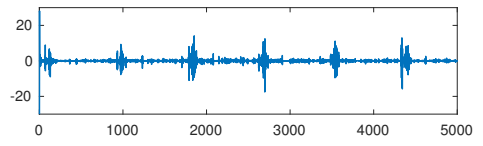

5

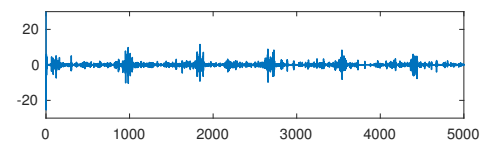

ธี

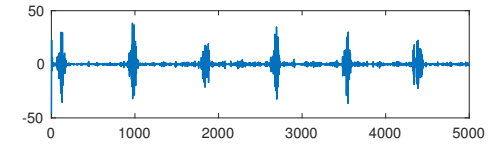

ธิ

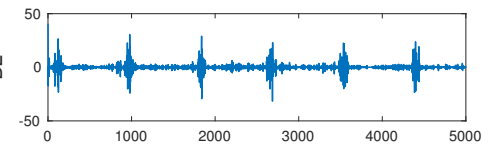

๓
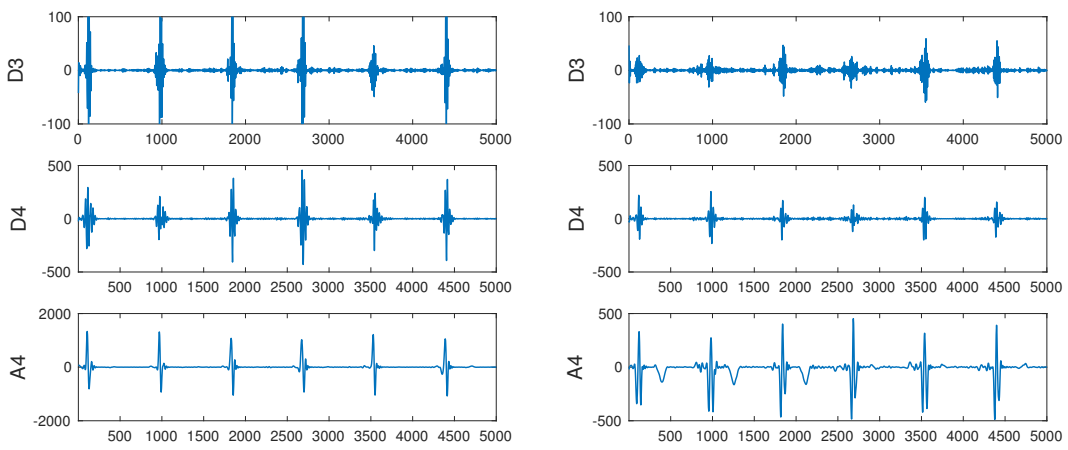
Samples

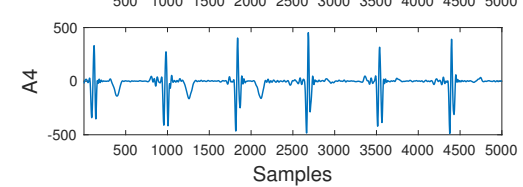

(c) Four levels DWT of $\mathrm{PRC}_{1}$ of the $V_{1}$ and $V_{2}$ vectors from MI. 

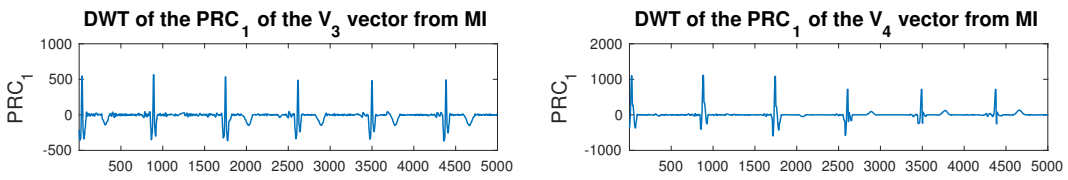

$\bar{\square}$
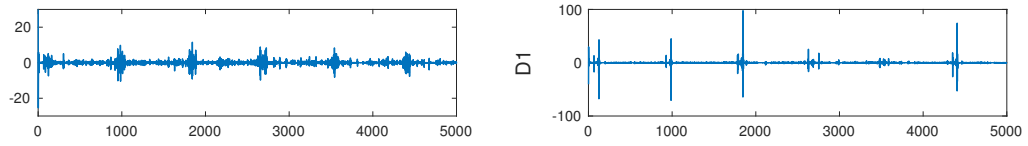

ธิ

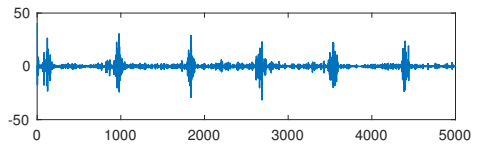

ธิ

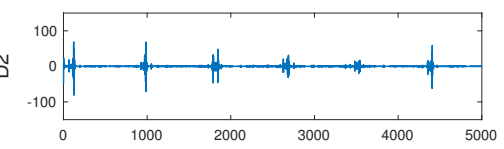

๓
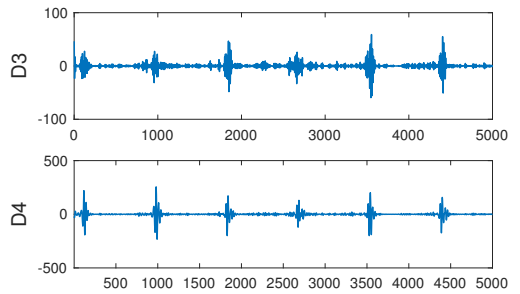

อ
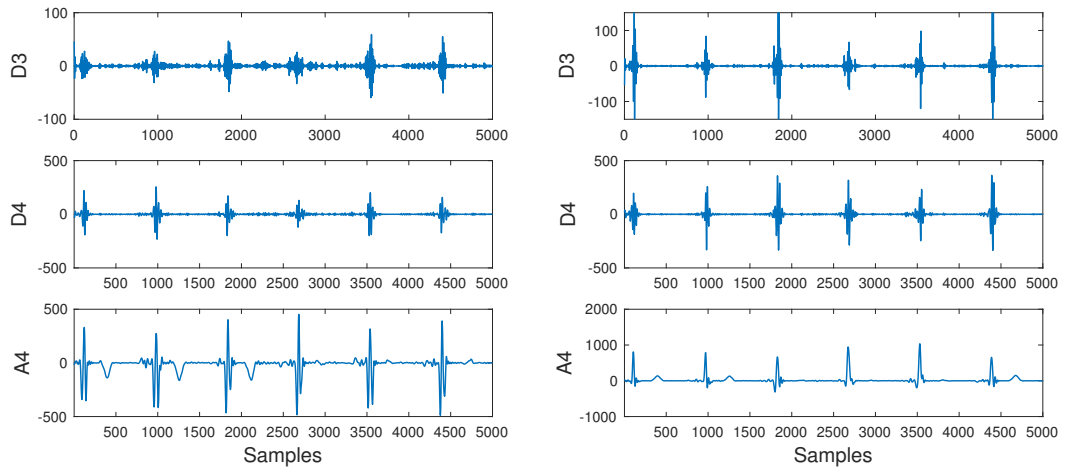

(d) Four levels DWT of $\mathrm{PRC}_{1}$ of the $V_{3}$ and $V_{4}$ vectors from MI.

Fig. 6 Samples of four levels DWT of $\mathrm{PRC}_{1}$ of the 4-dimensional cardiac vector: $V=\left[V_{1}, V_{2}, V_{3}, V_{4}\right]^{T}$ from $\mathrm{HC}$ and $\mathrm{Ml}$ subjects. 


\subsection{Phase space reconstruction (PSR)}

It is sometimes necessary to search for patterns in a time series and in a higher dimensional transformation of the time series [70]. Phase space reconstruction is a method used to reconstruct the so-called phase space. The concept of phase space is a useful tool for characterizing any low-dimensional or high-dimensional dynamic system. A dynamic system can be described using a phase space diagram, which essentially provides a coordinate system where the coordinates are all the variables comprising mathematical formulation of the system. A point in the phase space represents the state of the system at any given time [71, 72]. Every db3 wavelet function of the PRC of the cardiac vector can be written as the time series vector $V=\left\{v_{1}, v_{2}, v_{3}, \ldots, v_{K}\right\}$, where $K$ is the total number of data points. The phase space can be reconstructed according to [72]:

$$
Y_{j}=\left(V_{j}, V_{j+\tau}, V_{j+2 \tau}, \ldots, V_{j+(d-1) \tau}\right)
$$

where $j=1,2, \ldots, K-(d-1) \tau, d$ is the embedding dimension of the phase space and $\tau$ is a time lag. It is worthwhile to mention that the properties associated with the cardiac dynamics are preserved in the reconstructed phase space.

The behaviour of the signal over time can be visualized using PSR (especially when $d=2$ or 3 ). In this work, we have confined our discussion to the value of embedding dimension $d=3$, because of their visualization simplicity. In addition, different studies have found this value to best represent the attractor for human biological system [73, 74]. For $\tau$, we either use the first-zero crossing of the autocorrelation function for each time series or the average $\tau$ value obtained from all the time series in the training dataset using the method proposed in [75]. In this study, we consider the values of time lag $\tau=1$ to test the classification performance. PSR for $d=3$ has been referred to as 3D PSR.

Reconstructed phase spaces have been proven to be topologically equivalent to the original system and therefore are capable of recovering the nonlinear dynamics of the generating system $[76,77]$. This implies that the full dynamics of the cardiac system are accessible in this space, and for this reason, features extracted from it can potentially contain more and/or different information than the common features extraction method [78].

3D PSR is the plot of three delayed vectors $V_{j}, V_{j+1}$ and $V_{j+2}$ to visualize the dynamics of human cardiac system. Euclidian distance (ED) of a point $\left(V_{j}, V_{j+1}, V_{j+2}\right)$, which is the distance of the point from origin in 3D PSR and can be defined as [72]

$$
E D_{j}=\sqrt{V_{j}^{2}+V_{j+1}^{2}+V_{j+2}^{2}}
$$

ED measures can be used in features extraction and have been studied and applied in many fields, such as clustering algorithms and induced aggregation operators [79].

\subsection{Feature extraction and selection}

In order to obtain more efficient features, this paper proposes the following extraction scheme. 
(1) ITD of the cardiac vector and derivation of predominant PRCs. The signals obtained by ITD method, which are a series of decomposing signals, cannot be directly used to classify because of the high feature dimension. To solve this problem, the Pearson's correlation coefficient is calculated to measure the correlation between the first six PRCs and the original cardiac vector. The PRC component with higher correlation coefficient is more highly correlated to the original signal, which means the signal energy is mostly concentrated in this PRC as well. In the present study most of the energy is concentrated in $\mathrm{PRC}_{1}$ component, which have the most important information from the cardiac vector and are considered to be the predominant PRCs (seen from Table 3).

Table 3 The average correlation coefficients between each PRC and original cardiac vector from HC and $\mathrm{MI}$ subjects in the PTB database.

\begin{tabular}{lllllll}
\hline Cardiac vector type & \multicolumn{7}{c}{ Average correlation coefficients } \\
\cline { 2 - 7 } & $\mathrm{PRC}_{1}$ & $\mathrm{PRC}_{2}$ & $\mathrm{PRC}_{3}$ & $\mathrm{PRC}_{4}$ & $\mathrm{PRC}_{5}$ & $\mathrm{PRC}_{6}$ \\
\hline$V_{1}$ for HC & 0.6640 & 0.3671 & 0.3390 & 0.2873 & 0.1814 & 0.2889 \\
$V_{2}$ for HC & 0.4547 & 0.2704 & 0.2717 & 0.2233 & 0.2463 & 0.1556 \\
$V_{3}$ for HC & 0.7034 & 0.2865 & 0.3271 & 0.3165 & 0.1366 & 0.2643 \\
$V_{4}$ for $\mathrm{HC}$ & 0.6399 & 0.3025 & 0.2997 & 0.2633 & 0.1979 & 0.1732 \\
\hline \hline$V_{1}$ for $\mathrm{MI}$ & 0.5155 & 0.3262 & 0.3124 & 0.2474 & 0.2484 & 0.2086 \\
$V_{2}$ for $\mathrm{MI}$ & 0.5898 & 0.2809 & 0.2524 & 0.2498 & 0.2146 & 0.2073 \\
$V_{3}$ for $\mathrm{MI}$ & 0.6186 & 0.2647 & 0.2346 & 0.2153 & 0.2018 & 0.2034 \\
$V_{4}$ for $\mathrm{MI}$ & 0.5289 & 0.3190 & 0.2706 & 0.2478 & 0.2721 & 0.1972 \\
\hline \hline Mean & 0.5894 & 0.3021 & 0.2884 & 0.2563 & 0.2124 & 0.2123 \\
\hline
\end{tabular}

(2) Four levels DWT is employed to decompose the predominant PRCs into different frequency bands, in which third-order Daubechies (db3) wavelet function is selected for analysis. $\mathrm{D} 4$ of the $\mathrm{PRC}_{1}$ cardiac vector are regarded as reference variables $\left[V 1^{\mathrm{PRC}_{1}^{\mathrm{D} 4}}, V 2^{\mathrm{PRC}_{1}^{\mathrm{D} 4}}, V 3^{\mathrm{PRC}_{1}^{\mathrm{D} 4}}, V 4^{\mathrm{PRC}_{1}^{\mathrm{D} 4}}\right]^{T}$ and are used for feature derivation.

(3) Reconstruct the phase space of the reference variables with selected values of $d$ and $\tau$;

(4) Compute ED of 3D PSR of the reference variables. Concatenate them to form a feature vector $\left[E D_{j}^{V_{1}^{\mathrm{PRC}_{1}^{\mathrm{D}} 4}}, E D_{j}^{V_{2}^{\mathrm{PRC} 4}}, E D_{j}^{V_{3}^{\mathrm{PRC}_{1}^{\mathrm{D}} 4}}, E D_{j}^{V_{4}^{\mathrm{PRC}_{1}^{\mathrm{D}} 4}}\right]^{T}$.

For the PTB database, cardiac vectors are analyzed and cardiac system dynamics are extracted by using ITD, DWT and 3D PSR. First, ITD of the cardiac vector is exhibited in Figs. 4 and 5. Four levels DWT of the $\mathrm{PRC}_{1}$ of cardiac vector from the $\mathrm{HC}$ and MI subjects are demonstrated in Fig. 6. The db3 of the $\mathrm{PRC}_{1}$ is utilized to

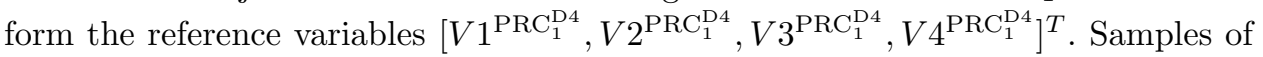
the 3D PSR of the reference variables are exhibited in Figs. 7 and 8. After 3D PSR,

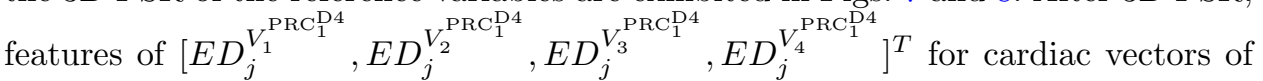
the $\mathrm{HC}$ and MI subjects are derived through ED computation, as demonstrated in Figs. 9 and 10. As we have analyzed before, significant difference in cardiac system dynamics have been reported between HC and MI subjects, which can also be seen obviously from Figs. 7 and 8 . 


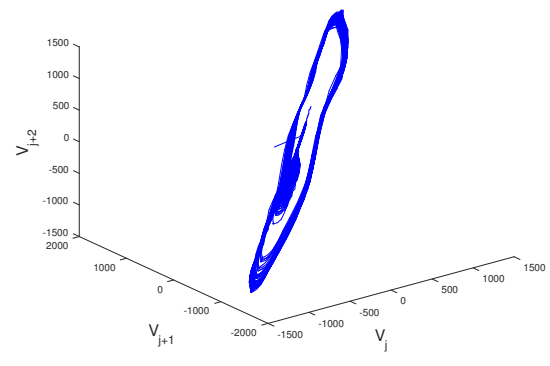

(a) 3D PSR of $\mathrm{PRC}_{1}^{\mathrm{D} 4}$ for $V_{1}$.

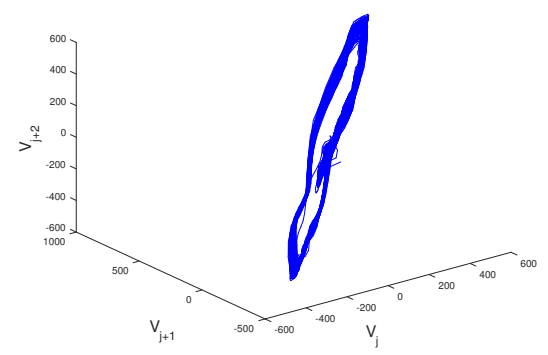

(c) 3D PSR of $\mathrm{PRC}_{1}^{\mathrm{D} 4}$ for $V_{3}$.

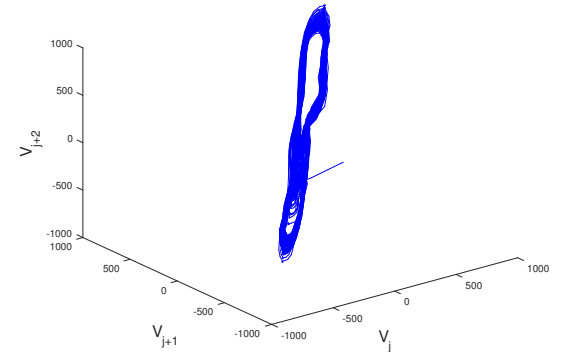

(b) $3 \mathrm{D}$ PSR of $\mathrm{PRC}_{1}^{\mathrm{D} 4}$ for $V_{2}$.

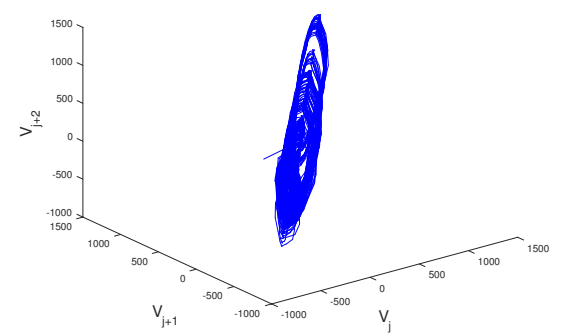

(d) 3D PSR of $\mathrm{PRC}_{1}^{\mathrm{D} 4}$ for $V_{4}$.

Fig. 7 Samples of 3D PSR of $\mathrm{PRC}_{1}^{\mathrm{D} 4}$ of 4-dimensional cardiac vector: $V=\left[V_{1}, V_{2}, V_{3}, V_{4}\right]^{T}$ from HC subject. 


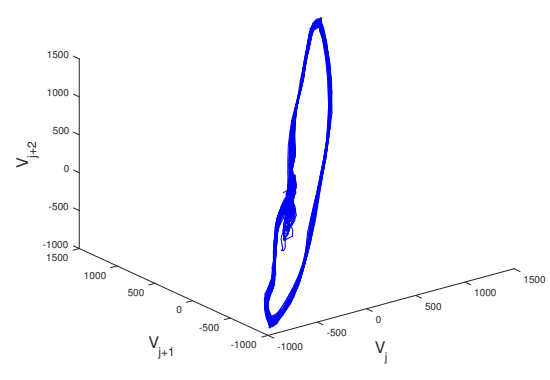

(a) 3D PSR of $\mathrm{PRC}_{1}^{\mathrm{D} 4}$ for $V_{1}$.

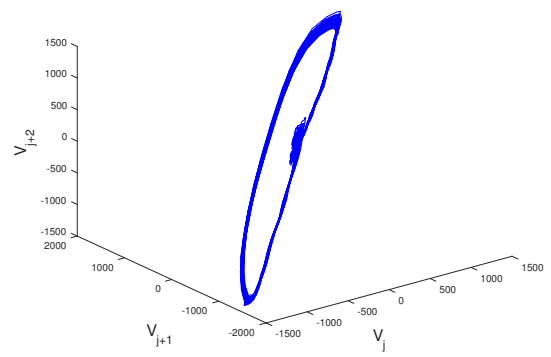

(c) 3D PSR of $\mathrm{PRC}_{1}^{\mathrm{D} 4}$ for $V_{3}$.

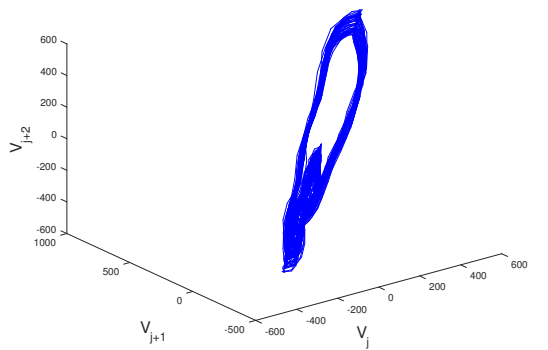

(b) 3D PSR of $\mathrm{PRC}_{1}^{\mathrm{D} 4}$ for $V_{2}$.

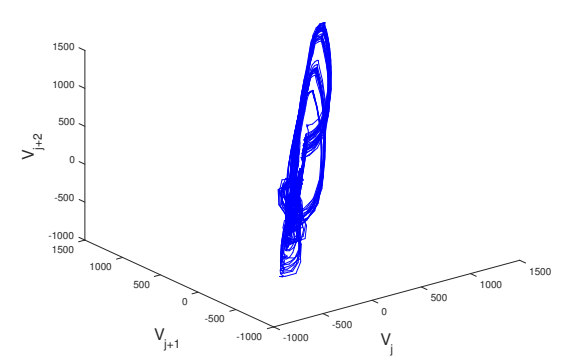

(d) 3D PSR of $\mathrm{PRC}_{1}^{\mathrm{D} 4}$ for $V_{4}$.

Fig. 8 Samples of 3D PSR of $\mathrm{PRC}_{1}^{\mathrm{D} 4}$ of 4-dimensional cardiac vector: $V=\left[V_{1}, V_{2}, V_{3}, V_{4}\right]^{T}$ from MI subject. 

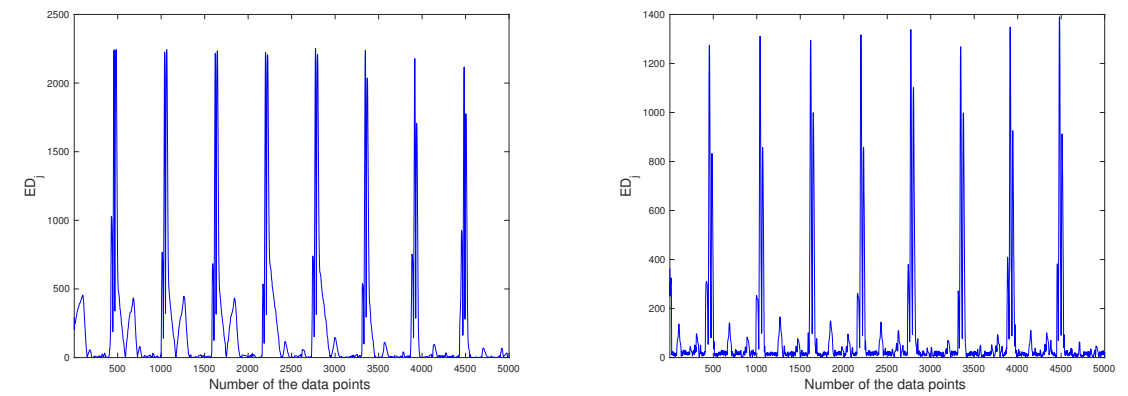

(a) Euclidian distance of 3D PSR of $\mathrm{PRC}_{1}^{\mathrm{D} 4}$ (b) Euclidian distance of 3D PSR of PRC ${ }_{1}^{\mathrm{D} 4}$ for $V_{1}$. for $V_{2}$.
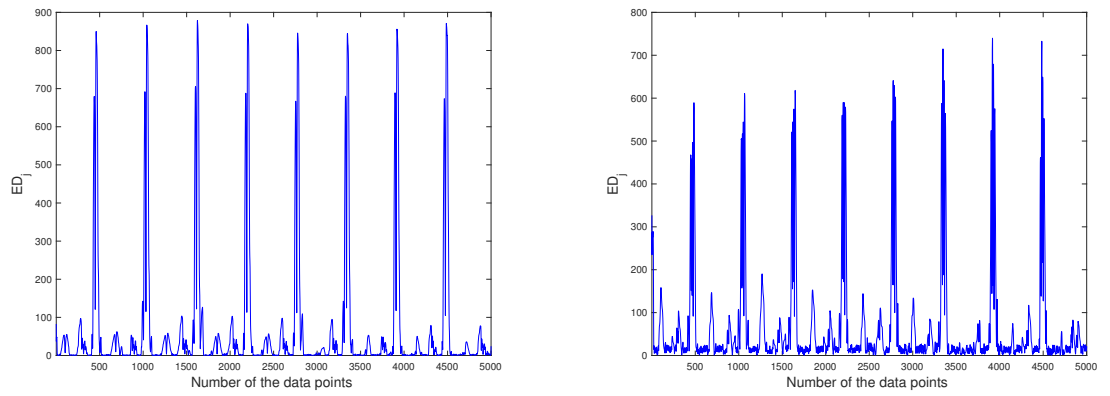

(c) Euclidian distance of 3D PSR of $\mathrm{PRC}_{1}^{\mathrm{D} 4}$ (d) Euclidian distance of 3D PSR of $\mathrm{PRC}_{1}^{\mathrm{D} 4}$ for $V_{3}$. for $V_{4}$.

Fig. 9 Samples of the Euclidian distance of 3D PSR of $\mathrm{PRC}_{1}^{\mathrm{D} 4}$ of 4-dimensional cardiac vector: $V=\left[V_{1}, V_{2}, V_{3}, V_{4}\right]^{T}$ from $\mathrm{HC}$ subject. 

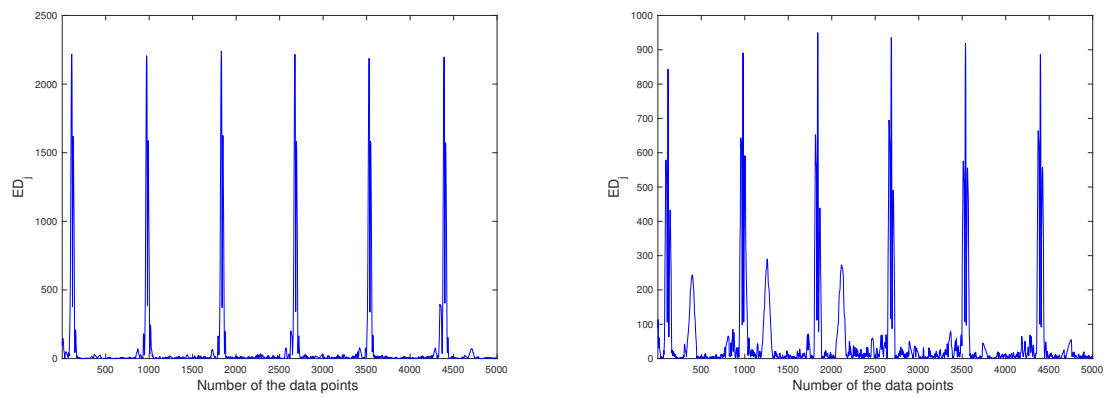

(a) Euclidian distance of 3D PSR of $\mathrm{PRC}_{1}^{\mathrm{D} 4}$ (b) Euclidian distance of 3D PSR of $\mathrm{PRC}_{1}^{\mathrm{D} 4}$ for $V_{1}$. for $V_{2}$.
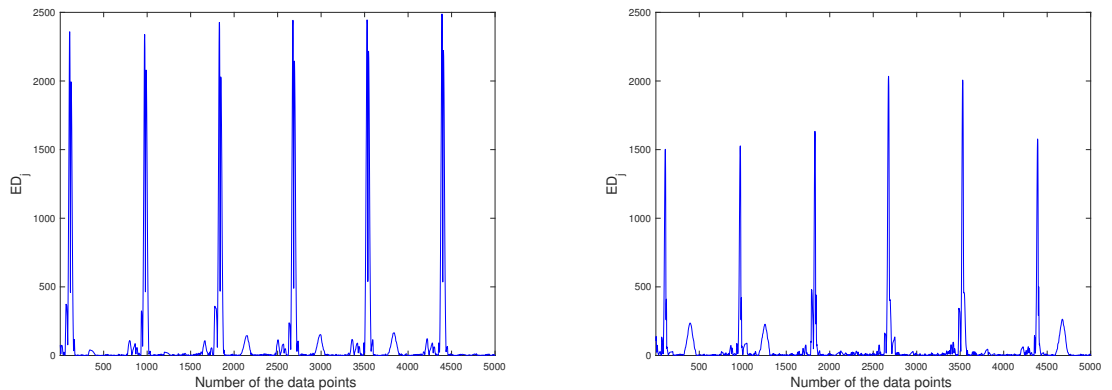

(c) Euclidian distance of 3D PSR of $\mathrm{PRC}_{1}^{\mathrm{D} 4}$ (d) Euclidian distance of 3D PSR of $\mathrm{PRC}_{1}^{\mathrm{D} 4}$ for $V_{3}$. for $V_{4}$.

Fig. 10 Samples of the Euclidian distance of 3D PSR of $\mathrm{PRC}_{1}^{\mathrm{D} 4}$ of 4-dimensional cardiac vector: $V=\left[V_{1}, V_{2}, V_{3}, V_{4}\right]^{T}$ from $\mathrm{Ml}$ subject. 


\subsection{Training and modeling mechanism based on selected features}

In this section, we present a scheme for modeling and derivation of nonlinear cardiac system dynamics derived from the synthesis of 12-leand and Frank XYZ leads from normal and MI subjects based on the extracted features.

Consider a general nonlinear cardiac system dynamics in the following form:

$$
\dot{x}=F(x ; p)+v(x ; p)
$$

where $x=\left[x_{1}, \ldots, x_{n}\right]^{T} \in R^{n}$ are the system states which represent the features $\left[E D_{j}^{V_{1}^{\mathrm{PRC}_{1}^{\mathrm{D}} 4}}, E D_{j}^{V_{2}^{\mathrm{PRC}_{1}}}, E D_{j}^{V_{3}^{\mathrm{PRC}_{1}^{\mathrm{D}} 4}}, E D_{j}^{V_{4}^{\mathrm{PRC}_{1} 4}}\right]^{T}$ is a constant vector of system parameters. $F(x ; p)=\left[f_{1}(x ; p), \ldots, f_{n}(x ; p)\right]^{T}$ is a smooth but unknown nonlinear vector representing the cardiac system dynamics, $v(x ; p)$ is the modeling uncertainty. Since the modeling uncertainty $v(x ; p)$ and the cardiac system dynamics $F(x ; p)$ cannot be decoupled from each other, we consider the two terms together as an undivided term, and define $\phi(x ; p):=F(x ; p)+v(x ; p)$ as the general cardiac system dynamics. Then, the following steps are taken to model and derive the cardiac system dynamics via deterministic learning theory [80-82].

In the first step, standard RBF neural networks are constructed in the following form

$$
f_{n n}(Z)=\sum_{i=1}^{N} w_{i} s_{i}(Z)=W^{T} S(Z),
$$

where $Z$ is the input vector, $W=\left[w_{1}, \ldots, w_{N}\right]^{T} \in R^{N}$ is the weight vector, $N$ is the node number of the neural networks, and $S(Z)=\left[s_{1}\left(\left\|Z-\mu_{1}\right\|\right), \ldots, s_{N}(\|\right.$ $\left.\left.Z-\mu_{N} \|\right)\right]^{T}$, with $s_{i}\left(\left\|Z-\mu_{i}\right\|\right)=\exp \left[\frac{-\left(Z-\mu_{i}\right)^{T}\left(Z-\mu_{i}\right)}{\eta_{i}^{2}}\right]$ being a Gaussian function, $\mu_{i}(i=1, \ldots, N)$ being distinct points in state space, and $\eta_{i}$ being the width of the receptive field.

In the second step, the following dynamical RBF neural networks are employed to model and derive the cardiac system dynamics $\phi(x ; p)$ :

$$
\dot{\hat{x}}=-A(\hat{x}-x)+\hat{W}_{j}^{T} S_{j}(x)
$$

where $\hat{x}=\left[\hat{x}_{1}, \ldots, \hat{x}_{n}\right]$ is the state vector of the dynamical RBF neural networks, $A=\operatorname{diag}\left[a_{1}, \ldots, a_{n}\right]$ is a diagonal matrix, with $a_{i}>0$ being design constants, localized RBF neural networks $\hat{W}_{j}^{T} S_{j}(x)=\sum_{i=1}^{N} w_{i j} s_{i j}(x)$ are used to approximate the unknown $\phi(x ; p)$, where $\hat{W}_{j}=\left[w_{1 j}, \ldots, w_{N j}\right]^{T}, S_{j}=\left[s_{1 j}, \ldots, s_{N j}\right]^{T}$, for $j=$ $1, \ldots, n$.

The following law is used to update the neural weights

$$
\dot{\hat{W}}_{i}=\dot{\tilde{W}}_{i}=-\Gamma_{i} S_{i}(x) \tilde{x}_{i}-\sigma_{i} \Gamma_{i} \hat{W}_{i}
$$

where $\tilde{x}_{i}=\hat{x}_{i}-x_{i}, \tilde{W}_{i}=\hat{W}_{i}-W_{i}^{*}, W_{i}^{*}$ is the ideal constant weight vector such that $\phi_{i}(x ; p)=W_{i}^{* T} S_{i}(x)+\epsilon_{i}(x), \epsilon_{i}(x)<\epsilon^{*}$ represents the neural network modeling error, $\Gamma_{i}=\Gamma_{i}^{T}>0$, and $\sigma_{i}>0$ is a small value. 
With Eqs. (10)-(12), the derivative of the state estimation error $\tilde{x}_{i}$ satisfies

$$
\dot{\tilde{x}}_{i}=-a_{i} \tilde{x}_{i}+\hat{W}_{i}^{T} S_{i}(x)-\phi_{i}(x ; p)=-a_{i} \tilde{x}_{i}+\tilde{W}_{i}^{T} S_{i}(x)-\epsilon_{i}
$$

In the third step, by using the local approximation property of RBF neural networks, the overall system consisting of dynamical model (14) and the neural weight updating law (13) can be summarized into the following form in the region $\Omega_{\zeta}$

$$
\left[\begin{array}{c}
\dot{\tilde{x}}_{i} \\
\dot{\tilde{W}}_{\zeta i}
\end{array}\right]=\left[\begin{array}{cc}
-a_{i} & S_{\zeta i}(x)^{T} \\
-\Gamma_{\zeta i} S_{\zeta i}(x) & 0
\end{array}\right]\left[\begin{array}{c}
\tilde{x}_{i} \\
\tilde{W}_{\zeta i}
\end{array}\right]+\left[\begin{array}{c}
-\epsilon_{\zeta i} \\
-\sigma_{i} \Gamma_{\zeta i} \hat{W}_{\zeta i}
\end{array}\right]
$$

and

$$
\dot{\hat{W}}_{\bar{\zeta} i}=\dot{\tilde{W}}_{\bar{\zeta}_{i}}=-\Gamma_{\bar{\zeta} i} S_{\bar{\zeta} i}(x) \tilde{x}_{i}-\sigma_{i} \Gamma_{\bar{\zeta} i} \hat{W}_{\bar{\zeta} i}
$$

where $\epsilon_{\zeta i}=\epsilon_{i}-\tilde{W}_{\bar{\zeta} i}^{T} S_{\bar{\zeta}}(x)$. The subscripts $(\cdot)_{\zeta}$ and $(\cdot)_{\bar{\zeta}}$ are used to stand for terms related to the regions close to and far away from the trajectory $\varphi_{\zeta}\left(x_{0}\right)$. The region close to the trajectory is defined as $\Omega_{\zeta}:=\left\{Z \mid \operatorname{dist}\left(Z, \varphi_{\zeta}\right) \leq d_{\iota}\right\}$, where $Z=x, d_{\iota}>0$ is a constant satisfying $s\left(d_{\iota}\right)>\iota, s(\cdot)$ is the RBF used in the network, $\iota$ is a small positive constant. The related subvectors are given as: $S_{\zeta i}(x)=$ $\left[s_{j 1}(x), \ldots, s_{j \zeta}(x)\right]^{T} \in R^{N_{\zeta}}$, with the neurons centered in the local region $\Omega_{\zeta}$, and $W_{\zeta}^{*}=\left[w_{j 1}^{*}, \ldots, w_{j \zeta}^{*}\right]^{T} \in R^{N_{\zeta}}$ is the corresponding weight subvector, with $N_{\zeta}<N$. For localized RBF neural networks, $\left|\tilde{W}_{\bar{\zeta} i}^{T} S_{\overline{\zeta i}}(x)\right|$ is small, so $\epsilon_{\zeta i}=O\left(\epsilon_{i}\right)$.

By the convergence result, we can obtain a constant vector of neural weights according to

$$
\bar{W}_{i}=\operatorname{mean}_{t \in\left[t_{a}, t_{b}\right]} \hat{W}_{i}(t)
$$

where $t_{b}>t_{a}>0$ represent a time segment after the transient process. Therefore, we conclude that accurate identification of the function $\phi_{i}(x ; p)$ is obtained along the trajectory $\varphi_{\zeta}\left(x_{0}\right)$ by using $\bar{W}_{i}^{T} S_{i}(x)$, i.e.,

$$
\phi_{i}(x ; p)=\bar{W}_{i}^{T} S_{i}(x)+\epsilon_{i 2}
$$

where $\epsilon_{i 2}=O\left(\epsilon_{i 1}\right)$ and subsequently $\epsilon_{i 2}=O\left(\epsilon^{*}\right)$.

\subsection{Classification mechanism}

In this section, we present a scheme to classify normal and MI cardiac vectors.

Consider a training dataset consisting of cardiac system patterns $\varphi_{\zeta}^{k}, k=$ $1, \ldots, M$, with the $k t h$ training pattern $\varphi_{\zeta}^{k}$ generated from

$$
\dot{x}=F^{k}\left(x ; p^{k}\right)+v^{k}\left(x ; p^{k}\right), \quad x\left(t_{0}\right)=x_{\zeta 0}
$$

where $F^{k}\left(x ; p^{k}\right)$ denotes the cardiac system dynamics, $v^{k}\left(x ; p^{k}\right)$ denotes the modeling uncertainty, $p^{k}$ is the system parameter vector. 
As demonstrated in Subsection 2.7, the general cardiac system dynamics $\phi^{k}\left(x ; p^{k}\right):=F^{k}\left(x ; p^{k}\right)+v^{k}\left(x ; p^{k}\right)$ can be accurately derived and preserved in constant RBF neural networks $\bar{W}^{k^{T}} S(x)$. By utilizing the learned knowledge obtained in the training stage, a bank of $M$ estimators is constructed for the training cardiac system patterns as follows:

$$
\dot{\bar{\chi}}^{k}=-B\left(\bar{\chi}^{k}-x\right)+\bar{W}^{k^{T}} S(x)
$$

where $k=1, \ldots, M$ is used to stand for the $k$ th estimator, $\bar{\chi}^{k}=\left[\bar{\chi}_{1}^{k}, \ldots, \bar{\chi}_{n}^{k}\right]^{T}$ is the state of the estimator, $B=\operatorname{diag}\left[b_{1}, \ldots, b_{n}\right]$ is a diagonal matrix which is kept the same for all estimators, $x$ is the state of an input test cardiac system pattern generated from Eq. (10).

In the classification phase, by comparing the test cardiac system pattern (standing for a $\mathrm{HC}$ or MI cardiac system pattern) generated from the cardiac system (10) with the set of $M$ estimators (20), we obtain the following test error systems:

$$
\dot{\tilde{\chi}}_{i}^{k}=-b_{i} \tilde{\chi}_{i}^{k}+\bar{W}_{i}^{k^{T}} S_{i}(x)-\phi_{i}(x ; p), \quad i=1, \ldots, n, \quad k=1, \ldots, M
$$

where $\tilde{\chi}_{i}^{k}=\bar{\chi}_{i}^{k}-x_{i}$ is the state estimation (or synchronization) error. We compute the average $L_{1}$ norm of the error $\tilde{\chi}_{i}^{k}(t)$

$$
\left\|\tilde{\chi}_{i}^{k}(t)\right\|_{1}=\frac{1}{\mathrm{~T}_{c}} \int_{t-\mathrm{T}_{c}}^{t}\left|\tilde{\chi}_{i}^{k}(\tau)\right| d \tau, \quad t \geq \mathrm{T}_{c}
$$

where $\mathrm{T}_{c}$ is the cycle of cardiac vector.

The fundamental idea of the classification between $\mathrm{HC}$ and MI cardiac vectors is that if a test cardiac system pattern is similar to the trained cardiac system pattern $s(s \in\{1, \ldots, k\})$, the constant RBF network $\bar{W}_{i}^{s^{T}} S_{i}(x)$ embedded in the matched estimator $s$ will quickly recall the learned knowledge by providing accurate approximation to the cardiac system dynamics. Thus, the corresponding error $\left\|\tilde{\chi}_{i}^{s}(t)\right\|_{1}$ will become the smallest among all the errors $\left\|\tilde{\chi}_{i}^{k}(t)\right\|_{1}$. Based on the smallest error principle, the appearing test cardiac system pattern can be classified. We have the following classification scheme.

Classification scheme: If there exists some finite time $t^{s}, s \in\{1, \ldots, k\}$ and some $i \in\{1, \ldots, n\}$ such that $\left\|\tilde{\chi}_{i}^{s}(t)\right\|_{1}<\left\|\tilde{\chi}_{i}^{k}(t)\right\|_{1}$ for all $t>t^{s}$, then the appearing cardiac system pattern can be classified and MI can be detected.

\section{Results}

Experiments are implemented using matlab software and tested on an Intel Core i7 $6700 \mathrm{~K} 3.5 \mathrm{GHz}$ computer with 64GB RAM. We assign feature vector sequences for all the heart beat records of $\mathrm{HC}$ and MI subjects in the PTB database. According to the method described in Subsection 2.6, we extract features, which means the input

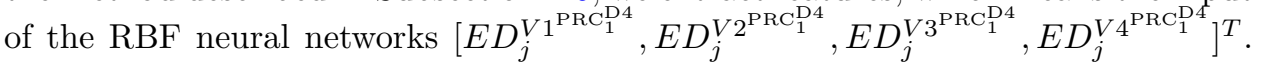
In order to eliminate data difference between different features, all feature data are normalized to $[-1,1]$.

Several experiments are carried out to verify the effectiveness of the proposed method on the $78 \mathrm{HC}$ and $367 \mathrm{MI}$ heart beat records. The classification results will 
be evaluated with the 10 -fold cross-validation style in which the variance of the estimate for the classifiers is reduced. The data are divided into the training and test subsets. For the 10-fold cross-validation, the data set is divided into ten subsets. Each time, one of the ten subsets is used as the test set and the other night subsets are put together to form a training set. As such, every fold has been used nine times as train data and one time as test data. The final result is the average of the 10 implementations. For the evaluation, six performance parameters are used including the Sensitivity (SEN), the Specificity (SPF), the Accuracy (ACC), the Positive Predictive Value (PPV), the Negative Predictive Value (NPV) and the Matthews Correlation Coefficient (MCC). These measurements are defined as follows [83]:

$$
\begin{aligned}
& \mathrm{SEN}=\frac{\mathrm{TP}}{\mathrm{TP}+\mathrm{FN}} \times 100(\%) \\
& \mathrm{SPF}=\frac{\mathrm{TN}}{\mathrm{TN}+\mathrm{FP}} \times 100(\%) \\
& \mathrm{ACC}=\frac{\mathrm{TP}+\mathrm{TN}}{\mathrm{TP}+\mathrm{TN}+\mathrm{FN}+\mathrm{FP}} \times 100(\%) \\
& \mathrm{PPV}=\frac{\mathrm{TP}}{\mathrm{TP}+\mathrm{FP}} \times 100(\%) \\
& \mathrm{NPV}=\frac{\mathrm{TN}}{\mathrm{TN}+\mathrm{FN}} \times 100(\%) \\
& \mathrm{MCC}=\frac{\mathrm{TP} \times \mathrm{TN}-\mathrm{FN} \times \mathrm{FP}}{\sqrt{(\mathrm{TP}+\mathrm{FN})(\mathrm{TP}+\mathrm{FP})(\mathrm{TN}+\mathrm{FN})(\mathrm{TN}+\mathrm{FP})}}
\end{aligned}
$$

where TP is the number of true positives, $\mathrm{FN}$ is the number of false negatives, $\mathrm{TN}$ is the number of true negatives and FP is the number of false positives. To be accurate, a classifier must have a high classification accuracy, a high sensitivity, as well as a high specificity [84]. For a larger value of MCC, the classifier performance will be better $[83,85]$.

The classification results on $\mathrm{HC}$ an MI subjects have been illustrated in Table 4 with 10-foldcross-validation style. Our study demonstrates the accuracy improvements to differentiate between HC and MI. Overall, our classification approach achieves good performance, which indicates that the proposed pattern recognition system can effectively classify MI by using nonlinear features and neural network based classification tools. 
Table 4 Performance of the proposed classification approach evaluated by 10 -fold cross-validation method.

\begin{tabular}{cccccccccc}
\hline Evaluation methods & Predicted groups & \multicolumn{2}{c}{ Actual groups } & SEN (\%) & SPF (\%) & ACC (\%) & PPV (\%) & NPV (\%) & MCC \\
\cline { 2 - 6 } & & HC & MI & & & & & & \\
\hline 10-fold cross-validation & HC & 76 & 2 & 98.37 & 97.44 & 98.20 & 99.45 & 92.68 & 0.9395 \\
& MI & 6 & 361 & & & & & \\
\hline
\end{tabular}

\section{Discussion}

Experimental results of this study revealed that MI subjects could be differentiated from $\mathrm{HC}$ subjects automatically by means of nonlinear features and neural networks based upon hybrid signal processing methods. The proposed scheme focuses not only on providing evidence to support the claim that MI subjects demonstrate altered cardiac system dynamics in comparison to $\mathrm{HC}$ subjects, but also on providing an automatic and objective method to detect MI cardiac vectors.

Banerjee and Mitra [22] employed cross wavelet transform (XWT) to produce wavelet cross spectrum (WCS) and wavelet coherence (WCOH) for the analysis of spectral differences between $\mathrm{HC}$ and MI subjects. The reported accuracy with threshold based classifier was $97.6 \%$ based on lead-III ECG signals. Arif et al. [86] extracted 117-dimensional features from ST-T region and Q wave region of each beat by using lead-wise principal components analysis (PCA) . Features were derived from 12-lead ECG signals and fed into the back propagation neural networks (BPNN) classifier for classification, which was with the reported accuracy of $93.7 \%$. Al-Kindiet et al. [87] used R-complex time index detection method to extract features from Q, R, S waves and $\mathrm{J}$ points, which could serve as an clinical indicator of MI. 12-lead ECG signals were derived from 20 normal and 20 MI subjects and the reported sensitivity was $85 \%$ for the detection of MI subjects. Liu et al. [88] fitted a given ECG signal with a 20th order polynomial function to derive new ECG features. They reported $94.4 \%$ classification accuracy with J48 decision tree model for the diagnosis of MI. Sharma et al. [89] calculated multiscale wavelet energies and eigenvalues of multiscale covariance matrices to act as as diagnostic features. The reported accuracy with the SVM RBF kernel classifier was $96 \%$. Sharma and Sunkaria [90] calculated sample entropy, normalized sub-band energy, log energy entropy and median slope over selected bands of 6 -lead ECG signals. These vectors were used as features and fed to the SVM classifier for the classification of MI and HC ECG signals. The reported accuracy was $98.84 \%$. Acharya et al. [91] constructed an end-to-end learning structure without any hand-crafted feature extraction by using a single lead ECG signal. The reported accuracy for the automated detection of normal and MI ECG beats (with noise and without noise) by using deep learning algorithm (CNN) was $93.53 \%$ and $95.22 \%$, respectively. Strodthoff and Strodthoff [92] also built an end-to-end learning structure without any hand-crafted feature extraction by including different combinations of leads, which could estimate the relative amount of information that these channels contribute to the classification decision. The reported accuracy for the automated detection of normal and MI ECG beatsby using CNN was $96.1 \%$. Lih et al. [93] proposed combined 16-layer CNN and Long Short-Term Memory (LSTM) models for the detection of MI with automatic feature extraction and selection. The reported accuracy was $98 \%$.

Different from the above discussed methods, this study proposes a hybrid method to extract nonlinear features using ITD, DWT, PSR and ED tools based on the 
synthesis of traditional 12-lead and Frank XYZ leads. These features are fed into dynamical estimators which are consisting of constant RBF neural networks to classify MI and HC subjects. Comparison of the classification performance to other state-of-the-art methods on the same database is demonstrated in Table 5. The proposed method provides an average classification accuracy of $98.20 \%$ through 10 -fold cross-validation style. Due to the use of 10 -fold cross-validation, the classification performance is robust. The method studied in this paper has the potential to serve as a supportive technical means to other approaches such as coronary arteriography and cardiac ultrasound for the diagnosis of MI. Although our classification accuracy is not higher than that reported in [90], we present a new classification tool together with building a novel feature vector rather than using directly the ECG signals. In addition, we used 4 features which are less than the 10 features used in [90]. Modeling, identification and classification of cardiac system dynamics were employed instead of putting feature vectors directly into the classifier in comparison to other methods. This provides another candidate tool for the detection of MI.

Table 5 Summary of classification performance on the HC and MI subjects obtained from the same PTB database in the literature.

\begin{tabular}{|c|c|c|c|c|c|}
\hline 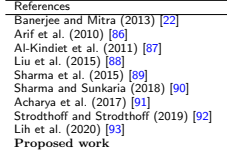 & $\begin{array}{l}\text { One lead (lead III) } \\
12 \text { leads } \\
12 \text { leads } \\
12 \text { leads } \\
12 \text { leads } \\
6 \text { leads } \\
\text { One lead (lead II) } \\
15 \text { leads } \\
\text { One lead (lead II) } \\
15 \text { leads }\end{array}$ & $\begin{array}{l}\text { ve } \\
\text { ts }\end{array}$ & $\begin{array}{l}\text { assifiers } \\
\text { rierhold based classifier } \\
\text { ck propagation neural netw } \\
\text { th mentioned } \\
8 \text { decision tree } \\
\text { lM with RBF kernel } \\
\text { JM } \\
\text { NN } \\
\text { NN } \\
\text { vN-LSTM } \\
\text { ynamical estimators }\end{array}$ & $\begin{array}{l}\text { la-fol } \\
\text { No } \\
\text { No } \\
\text { No } \\
\text { No }\end{array}$ & $\begin{array}{l}=97.6 \% \\
=93.7 \% \\
=85 \% \\
=93.7 \% \\
==96 \% \\
=98.84 \% \\
=09.22 \% \\
=99.1 \% \\
=98 \% \\
=y=98.20 \%\end{array}$ \\
\hline
\end{tabular}

\section{Conclusions}

In this study, a novel feature extraction technique including ITD, DWT, PSR and ED is proposed for detection of myocardial infarction, which is computationally simple and easy to implement. The results of this study indicate that the pattern classification of cardiac vector based on the synthesis of traditional 12-lead and Frank XYZ leads can offer an objective method to assess the disparity of cardiac system dynamics between HC and MI subjects. Synthesis of 12-lead and Frank XYZ leads to build a new 4-dimensional cardiac vector may fully reflect the pathological alterations in the abnormal patterns of cardiac system dynamics provoked by MI . ITD could extract most important information of the cardiac vector through predominant PRCs. DWT decomposes the predominant PRCs into different frequency bands, which are used to construct the reference variables. PSR plots cardiac system dynamics along the advisable db3 sub-bands (D4) of predominant PRCs trajectory in a 3D phase space diagram and visualizes the cardiac system dynamics. ED measures and derives features, which are fed into RBF neural networks for the modeling, identification and classification of cardiac system dynamics between $\mathrm{HC}$ and MI subjects. However, some limitations such as the relatively small size of the database, the regulation principle of the embedding dimension and time lag, still need to be improved and overcome. Future work will include a clinical validation of the proposed technique with a larger number of patients. Assessments of the relationship between the embedding dimension, time lag and the classification accuracy can also be considered in future investigations. It would be of interest to develop strategy for adaptive selection of PSR parameters which could create best classification performance. Features introduced in other methods such as various entropies, Hurst exponent, fractal dimension and other nonlinear features, can also be 
explored in the proposed framework to evaluate its classification performance. The proposed automated MI detection system can assist cardiologists in cross-checking their diagnosis. In addition, it can be extended to grade the severity of MI.

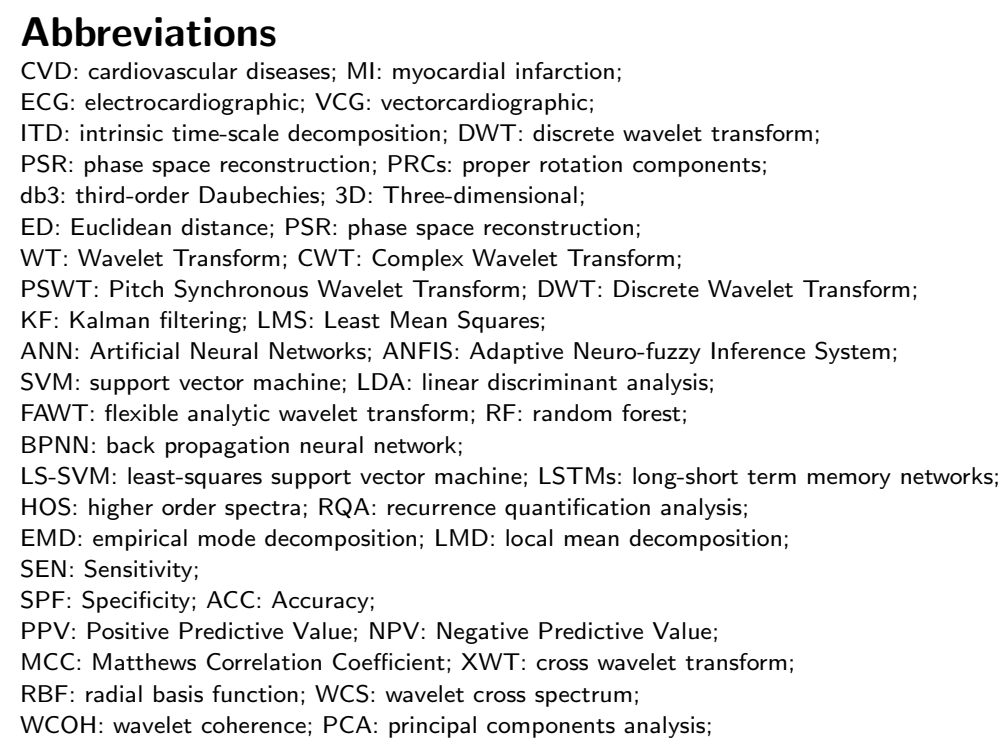

\section{Declarations}

Ethical approval and consent to participate

Not applicable.

Consent for publication

Not applicable.

Availability of data and materials

The datasets used and/or analyzed during in current study are available from the corresponding author on reasonable requests.

Competing interests

The authors declare that they have no competing interests.

Funding

This work was supported by the National Natural Science Foundation of China (Grant no. 61773194), by the Natural Science Foundation of Fujian Province (Grant no. 2018J01542), and by the Training Program of Innovation and Entrepreneurship for Undergraduates (Grant no. 202011312001).

Author's contributions

Study concept and design (WZ); drafting of the manuscript (WZ, ZL and CY); critical revision of the manuscript for important intellectual content (WZ and CY); obtained funding (WZ); administrative, technical, and material support (WZ); study supervision (WZ). All authors read and approved the final manuscript.

Acknowledgements

Not applicable.

Author details

${ }^{1}$ School of Physics and Mechanical and Electrical Engineering, Longyan University, Longyan 364012, China. ${ }^{2}$ Department of Mechanical, Industrial and Systems Engineering, University of Rhode Island, Kingston, RI 02881, USA.

References

1. Maheshwari S, Acharyya A, Schiariti M, Puddu PE (2015) Personalized reduced 3-lead system formation methodology for remote health monitoring applications and reconstruction of standard 12-lead system. International Archives of Medicine 8.

2. Hejc J, Vitek M, Ronzhina M, Novakova M, Kolarova J (2015) A wavelet-based ECG delineation method: adaptation to an experimental electrograms with manifested global ischemia. Cardiovascular Engineering and Technology 6(3): 364-375 
3. Wehr G, Peters RJ, Khalife K, Banning AP, Kuehlkamp V, Rickards AF, Sechtem U (2006) A vector-based, 5-electrode, 12-lead monitoring ECG (EASI) is equivalent to conventional 12-lead ECG for diagnosis of acute coronary syndromes. Journal of Electrocardiology 39(1): 22-28.

4. Lee HJ, Lee DS, Kwon HB, Kim DY, Park KS (2017) Reconstruction of 12-lead ECG using a single-patch device. Methods of Information in Medicine 56(04): 319-327.

5. Sahoo P, Thakkar H, Lee MY (2017) A cardiac early warning system with multi channel SCG and ECG monitoring for mobile health. Sensors 17(4): 711.

6. Tomasic I, Trobec R (2013) Electrocardiographic systems with reduced numbers of leads-synthesis of the 12-lead ECG. IEEE Reviews in Biomedical Engineering 7: 126-142.

7. Scherer JA, Jenkins JM, Nicklas JM (1989) Synthesis of the 12-lead electrocardiogram from a 3-lead subset using patient-specific transformation vectors. An algorithmic approach to computerized signal synthesis. Journal of Electrocardiology 22: 122-128.

8. Nelwan SP, Kors JA, Meij SH (2000) Minimal lead sets for reconstruction of 12-lead electrocardiograms. Journal of Electrocardiology 33: 163-166.

9. Nelwan SP, Kors JA, Meij SH, van Bemmel JH, Simoons ML (2004) Reconstruction of the 12-lead electrocardiogram from reduced lead sets. Journal of Electrocardiology 37(1): 11-18.

10. Drew BJ, Pelter MM, Brodnick DE, Yadav AV (2002) Comparison of a new reduced lead set ECG with the standard ECG for diagnosing cardiac arrhythmias and myocardial ischemia. Journal of Electrocardiology 35: 13

11. Atoui H, Fayn J, Rubel P (2010) A novel neural-network model for deriving standard 12-lead ECGs from serial three-lead ECGs: application to self-care. IEEE Transactions on Information Technology in Biomedicine 14(3): 883-890.

12. Nallikuzhy JJ, Dandapat S (2017) Spatial enhancement of ECG using diagnostic similarity score based lead selective multi-scale linear model. Computers in Biology and Medicine 85: 53-62.

13. Frank $E$. A direct experimental study of three systems of spatial vectorcardiography. Circulation 10: 101

14. Dawson D, Yang H, Malshe M, Bukkapatnam ST, Benjamin B, Komanduri R (2009) Linear affine transformations between 3-lead (Frank XYZ leads) vectorcardiogram and 12-lead electrocardiogram signals. Journal of Electrocardiology 42(6): 622-630.

15. Dower GE. A lead synthesizer for the Frank system to simulate the standard 12-lead electrocardiogram. Journal of Electrocardiology 1(1): 101-116

16. Maheshwari S, Acharyya A, Puddu PE, Mazomenos EB, Schiariti M, Maharatna K (2016) Robust and accurate personalised reconstruction of standard 12-lead system from Frank vectorcardiographic system. Computer Methods in Biomechanics and Biomedical Engineering: Imaging \& Visualization 4(3-4): 183-192.

17. Kim WS, Jin SH, Park YK, Choi HM (2007) A study on development of multi-parametric measure of heart rate variability diagnosing cardiovascular disease. In World Congress on Medical Physics and Biomedical Engineering, Springer, pp. 3480-3483.

18. Lee HG, Noh KY, Ryu KH (2007) Mining biosignal data: coronary artery disease diagnosis using linear and nonlinear features of HRV. In Pacific-Asia Conference on Knowledge Discovery and Data Mining, Springer, pp. 218-228.

19. Acharya UR, Sudarshan VK, Koh JE, Martis RJ, Tan JH, Oh SL, Chua CK (2017) Application of higher-order spectra for the characterization of coronary artery disease using electrocardiogram signals. Biomedical Signal Processing and Control 31: 31-43.

20. Babaoglu I, Findik O, Ulker E (2010) A comparison of feature selection models utilizing binary particle swarm optimization and genetic algorithm in determining coronary artery disease using support vector machine. Expert Systems with Applications 37(4): 3177-3183.

21. Kumar P, Sharma SK, Prasad S (2016) CAD for detection of fetal electrocardiogram by using wavelets and neuro-fuzzy systems. International Journal of Applied Engineering Research 11(4): 2321-2326.

22. Banerjee S, Mitra M (2013) Application of cross wavelet transform for ECG pattern analysis and classification. IEEE Transactions on Instrumentation and Measurement 63(2): 326-333.

23. Yochum M, Renaud C, Jacquir S (2016) Automatic detection of $P, Q R S$ and $T$ patterns in 12 leads ECG signal based on CWT. Biomedical Signal Processing and Control 25: 46-52.

24. Jagannath DJ, Selvakumar AI (2014) Issues and research on foetal electrocardiogram signal elicitation. Biomedical Signal Processing and Control 10: 224-244

25. Khambhati V, Patel MB (2017) Extraction of a respiration rate from ECG signal using discrete wavelet transform during exercise. Imperial Journal of Interdisciplinary Research 3(2): 1238-1241.

26. Chen S, Hua W, Li Z, Li J, Gao X (2017) Heartbeat classification using projected and dynamic features of ECG signal. Biomedical Signal Processing and Control 31: 165-173.

27. Roonizi EK, Sassi R (2015) A signal decomposition model-based Bayesian framework for ECG components separation. IEEE Transactions on Signal Processing 64(3): 665-674.

28. Kumar R, Kumar A, Singh GK (2016) Hybrid method based on singular value decomposition and embedded zero tree wavelet technique for ECG signal compression. Computer Methods and Programs in Biomedicine 129: 135-148.

29. Kumar SU, Inbarani HH (2017) Neighborhood rough set based ECG signal classification for diagnosis of cardiac diseases. Soft Computing 21(16): 4721-4733.

30. Shadmand S, Mashoufi B (2016) A new personalized ECG signal classification algorithm using block-based neural network and particle swarm optimization. Biomedical Signal Processing and Control 25: 12-23.

31. Homaeinezhad MR, Atyabi SA, Tavakkoli E, Toosi HN, Ghaffari A, Ebrahimpour R (2012) ECG arrhythmia recognition via a neuro-SVM-KNN hybrid classifier with virtual QRS image-based geometrical features. Expert Systems with Applications 39(2): 2047-2058.

32. Mathews SM, Kambhamettu C, Barner KE (2018) A novel application of deep learning for single-lead ECG classification. Computers in Biology and Medicine 99: 53-62.

33. Acharya UR, Fujita H, Lih OS, Adam M, Tan JH, Chua CK (2017) Automated detection of coronary artery disease using different durations of ECG segments with convolutional neural network. Knowledge-Based 
Systems 132: 62-71.

34. Acharya UR, Oh SL, Hagiwara Y, Tan JH, Adam M, Gertych A, Tan RS (2017) A deep convolutional neural network model to classify heartbeats. Computers in Biology and Medicine 89: 389-396.

35. Acharya UR, Fujita H, Lih OS, Hagiwara Y, Tan JH, Adam M (2017) Automated detection of arrhythmias using different intervals of tachycardia ECG segments with convolutional neural network. Information Sciences 405: 81-90.

36. Tan JH, Hagiwara Y, Pang W, Lim I, Oh SL, Adam M, Acharya UR (2018) Application of stacked convolutional and long short-term memory network for accurate identification of CAD ECG signals. Computers in Biology and Medicine 94: 19-26.

37. Yildirim O (2018) A novel wavelet sequence based on deep bidirectional LSTM network model for ECG signal classification. Computers in Biology and Medicine 96: 189202.

38. Baloglu UB, Talo M, Yildirim O, San-Tan R, Acharya UR (2019) Classification of myocardial infarction with multi-lead ECG signals and deep CNN. Pattern Recognition Letters 122: 23-30.

39. Jahmunah V, Oh SL, Wei JKE, Ciaccio EJ, Chua K, San TR, Acharya UR (2019) Computer-aided diagnosis of congestive heart failure using ECG signals-A review. Physica Medica 62: 95-104.

40. Sharma M, Acharya UR (2019) A new method to identify coronary artery disease with ECG signals and time-Frequency concentrated antisymmetric biorthogonal wavelet filter bank. Pattern Recognition Letters 125: 235-240.

41. Plawiak P, Acharya UR (2019) Novel deep genetic ensemble of classifiers for arrhythmia detection using ECG signals. Neural Computing and Applications, https://doi.org/10.1007/s00521-018-03980-2.

42. Bhurane AA, Sharma M, San-Tan R, Acharya UR (2019) An efficient detection of congestive heart failure using frequency localized filter banks for the diagnosis with ECG signals. Cognitive Systems Research 55: 82-94.

43. Varatharajan R, Manogaran G, Priyan MK (2018) A big data classification approach using LDA with an enhanced SVM method for ECG signals in cloud computing. Multimedia Tools and Applications 77(8): 10195-10215.

44. Plawiak P (2018) Novel methodology of cardiac health recognition based on ECG signals and evolutionary-neural system. Expert Systems with Applications 92: 334-349.

45. Kumar M, Pachori R, Acharya U (2017) Automated diagnosis of myocardial infarction ECG signals using sample entropy in flexible analytic wavelet transform framework. Entropy 19(9): 488.

46. Abawajy JH, Kelarev AV, Chowdhury M (2013) Multistage approach for clustering and classification of ECG data. Computer Methods and Programs in Biomedicine 112(3): 720-730.

47. Raj S, Ray KC (2017) ECG signal analysis using DCT-based DOST and PSO optimized SVM. IEEE Transactions on Instrumentation and Measurement 66(3): 470-478.

48. Acharya UR, Hagiwara Y, Koh JEW, Oh SL, Tan JH, Adam M, San Tan R (2018) Entropies for automated detection of coronary artery disease using ECG signals: A review. Biocybernetics and Biomedical Engineering 38(2): 373-384.

49. Ubeyli ED (2009) Adaptive neuro-fuzzy inference system for classification of ECG signals using Lyapunov exponents. Computer Methods and Programs in Biomedicine 93(3): 313-321.

50. Mishra AK, Raghav S (2010) Local fractal dimension based ECG arrhythmia classification. Biomedical Signal Processing and Control 5(2): 114-123.

51. Martis RJ, Acharya UR, Adeli H (2014) Current methods in electrocardiogram characterization. Computers in Biology and Medicine 48: 133-149.

52. Yang H (2010) Multiscale recurrence quantification analysis of spatial cardiac vectorcardiogram signals. IEEE Transactions on Biomedical Engineering 58(2): 339-347.

53. Bhaduri A, Ghosh D (2016) Quantitative assessment of heart rate dynamics during meditation: An ECG based study with multi-fractality and visibility graph. Frontiers in Physiology 7: 44

54. Sivakumar S, Nedumaran D (2018) Discrete time-frequency signal analysis and processing techniques for non-stationary signals. Journal of Applied Mathematics and Physics 6(09): 1916.

55. Huang NE, Shen Z, Long SR, Wu MC, Shih HH, Zheng Q, Liu HH (1998) The empirical mode decomposition and Hilbert spectrum for nonlinear and non-stationary time series analysis. Proceedings of the Royal Society of London A: Mathematical, Physical and Engineering Sciences. The Royal Society 454(1971): 903-995.

56. Huang B, Kunoth A (2013) An optimization based empirical mode decomposition scheme. Journal of Computational and Applied Mathematics 240: 174-183.

57. Park C, Looney D, Van Hulle MM, Mandic DP (2011) The complex local mean decomposition. Neurocomputing 74(6): 867-875.

58. Frei MG, Osorio I (2007) Intrinsic time-scale decomposition: time-frequency-energy analysis and real-time filtering of non-stationary signals. In Proceedings of the Royal Society of London A: Mathematical, Physical and Engineering Sciences (Vol. 463, No. 2078, pp. 321-342). The Royal Society.

59. Ghobadi Azbari P, Mohaqeqi S, Ghanbarzadeh Gashti N, Mikaili M (2016) Introducing a combined approach of empirical mode decomposition and PCA methods for maternal and fetal ECG signal processing. The Journal of Maternal-Fetal \& Neonatal Medicine 29(19): 3104-3109.

60. Lu L, Yan J, de Silva CW (2016) Feature selection for ECG signal processing using improved genetic algorithm and empirical mode decomposition. Measurement 94: 372-381.

61. Bousseljot R, Kreiseler D, Schnabel A (1995) Nutzung der EKG-Signaldatenbank CARDIODAT der PTB über das Internet. Biomedizinische Technik/Biomedical Engineering 40(s1): 317-318.

62. Goldberger AL, Amaral LA, Glass L, Hausdorff JM, Ivanov PC, Mark RG, Stanley HE (2000) PhysioBank, PhysioToolkit, and PhysioNet: components of a new research resource for complex physiologic signals. Circulation 101(23): e215-e220.

63. Dower GE, Machado HB (1979) XYZ data interpreted by a 12-lead computer program using the derived electrocardiogram. Journal of Electrocardiology 12(3): 249-261.

64. Kors JA, Van Herpen G, Sittig AC, Van Bemmel JH (1990) Reconstruction of the Frank vectorcardiogram from standard electrocardiographic leads: diagnostic comparison of different methods. European Heart Journal 
11(12): 1083-1092.

65. Feng Z, Lin X, Zuo MJ (2016) Joint amplitude and frequency demodulation analysis based on intrinsic time-scale decomposition for planetary gearbox fault diagnosis. Mechanical Systems and Signal Processing 72: 223-240.

66. Thakor NV, Webster JG, Tompkins WJ (1984) Estimation of QRS complex power spectra for design of a QRS filter. IEEE Transactions on Biomedical Engineering (11): 702-706.

67. Vavadi H, Ayatollahi A, Mirzaei A (2010) A wavelet-approximate entropy method for epileptic activity detection from EEG and its sub-bands. Journal of Biomedical Science and Engineering 3(12): 1182.

68. Tawfik NS, Youssef SM, Kholief M (2016) A hybrid automated detection of epileptic seizures in EEG records. Computers \& Electrical Engineering 53: 177-190.

69. Li M, Chen W, Zhang T (2017) Classification of epilepsy EEG signals using DWT-based envelope analysis and neural network ensemble. Biomedical Signal Processing and Control 31: 357-365.

70. Sun Y, Li J, Liu J, Chow C, Sun B, Wang R (2015) Using causal discovery for feature selection in multivariate numerical time series. Machine Learning 101(1-3): 377-395.

71. Sivakumar B (2002) A phase-space reconstruction approach to prediction of suspended sediment concentration in rivers. Journal of Hydrology 258 (1-4): 149-162.

72. Lee SH, Lim JS, Kim JK, Yang J, Lee Y (2014) Classification of normal and epileptic seizure EEG signals using wavelet transform, phase-space reconstruction, and Euclidean distance. Computer Methods and Programs in Biomedicine 116(1): 10-25.

73. Venkataraman $V$, Turaga $P(2016)$ Shape distributions of nonlinear dynamical systems for video-based inference. IEEE Transactions on Pattern Analysis and Machine Intelligence 38(12): 2531-2543.

74. Som A, Krishnamurthi N, Venkataraman V, Turaga P (2016) Attractor-shape descriptors for balance impairment assessment in Parkinson's disease. In IEEE Conference on Engineering in Medicine and Biology Society, pp. 3096-3100.

75. Michael S (2005) Applied nonlinear time series analysis: applications in physics, physiology and finance (Vol. 52). World Scientific.

76. Takens $F(1980)$ Detecting strange attractors in turbulence, in: Dynamical Systems and Turbulence, Warwick 1980, Springer, Berlin/Heidelberg, 1981, pp. 366-381.

77. Xu B, Jacquir S, Laurent G, Bilbault JM, Binczak S (2013) Phase space reconstruction of an experimental model of cardiac field potential in normal and arrhythmic conditions, in: 35th Annual International Conference of the IEEE Engineering in Medicine and Biology Society, pp. 3274-3277.

78. Chen M, Fang $Y$, Zheng $X$ (2014) Phase space reconstruction for improving the classification of single trial EEG. Biomedical Signal Processing and Control 11: 10-16.

79. Merigó JM, Casanovas M (2011) Induced aggregation operators in the Euclidean distance and its application in financial decision making. Expert Systems with Applications 38: 7603-7608.

80. Wang C, Hill DJ (2006) Learning from neural control. IEEE Transactions on Neural Networks 17(1): 130-146.

81. Wang C, Hill DJ (2007) Deterministic learning and rapid dynamical pattern recognition. IEEE Transactions on Neural Networks 18(3): 617-630.

82. Wang C, Hill DJ (2009) Deterministic Learning Theory for Identification, Recognition and Control. CRC Press, Boca Raton, FL.

83. Azar AT, El-Said SA (2014) Performance analysis of support vector machines classifiers in breast cancer mammography recognition. Neural Computing and Applications 24: 1163-1177.

84. Chu K (1999) An introduction to sensitivity, specificity, predictive values and likelihood ratios. Emergency Medicine Australasia 11(3): 175-181.

85. Yuan Q, Cai C, Xiao H, Liu X, Wen Y (2007) Diagnosis of breast tumours and evaluation of prognostic risk by using machine learning approaches. In D. S. Huang, L. Heutte, \& M. Loog (Eds.), Advanced intelligent computing theories and applications. With aspects of contemporary intelligent computing techniques (pp. 1250-1260). Springer.

86. Arif M, Malagore IA, Afsar FA (2010) Automatic detection and localization of myocardial infarction using back propagation neural networks. In Proceedings of the 4th International Conference on Bioinformatics and Biomedical Engineering, pp. 1-4.

87. Al-Kindi SG, Ali F, Farghaly A, Nathani M, Tafreshi R (2011) Towards real-time detection of myocardial infarction by digital analysis of electrocardiograms. In Proceedings of the 1st Middle East Conference on Biomedical Engineering, pp. 454-457.

88. Liu B, Liu J, Wang G, Huang K, Li F, Zheng Y, Zhou F (2015) A novel electrocardiogram parameterization algorithm and its application in myocardial infarction detection. Computers in Biology and Medicine 61: 178-184.

89. Sharma LN, Tripathy RK, Dandapat S (2015) Multiscale energy and eigenspace approach to detection and localization of myocardial infarction. IEEE Transactions on Biomedical Engineering 62(7): 1827-1837.

90. Sharma LD, Sunkaria RK (2018) Inferior myocardial infarction detection using stationary wavelet transform and machine learning approach. Signal, Image and Video Processing 12(2): 199-206.

91. Acharya UR, Fujita H, Oh SL, Hagiwara Y, Tan JH, Adam M (2017) Application of deep convolutional neural network for automated detection of myocardial infarction using ECG signals. Information Sciences 415: 190-198.

92. Strodthoff N, Strodthoff C (2019) Detecting and interpreting myocardial infarction using fully convolutional neural networks. Physiological Measurement 40(1): 015001.

93. Lih, OS, Jahmunah V, San TR, Ciaccio EJ, Yamakawa T, Tanabe M, Acharya UR (2020) Comprehensive electrocardiographic diagnosis based on deep learning. Artificial Intelligence in Medicine 103: 101789.

\section{Figure Legends}

Fig. 1. Flowchart of the proposed method for the MI classification using syntheses of lead system, ITD, DWT, PSR, ED and neural networks. 
Fig. 2. Examples of the synthesis of 4-dimensional cardiac vector: $V=\left[V_{1}, V_{2}, V_{3}, V_{4}\right]^{T}$ from $\mathrm{HC}$ subject.

Fig. 3. Examples of the synthesis of 4-dimensional cardiac vector: $V=\left[V_{1}, V_{2}, V_{3}, V_{4}\right]^{T}$ from $\mathrm{MI}$ subject.

Fig. 4. Samples of the ITD of 4-dimensional cardiac vector from HC subject.

Fig. 5. Samples of the ITD of 4-dimensional cardiac vector from MI subject.

Fig. 6. Samples of four levels DWT of $\mathrm{PRC}_{1}$ of the 4-dimensional cardiac vector: $V=\left[V_{1}, V_{2}, V_{3}, V_{4}\right]^{T}$ from $\mathrm{HC}$ and $\mathrm{MI}$ subjects.

Fig. 7. Samples of 3D PSR of $\mathrm{PRC}_{1}^{\mathrm{D} 4}$ of 4-dimensional cardiac vector: $V=\left[V_{1}, V_{2}, V_{3}, V_{4}\right]^{T}$ from $\mathrm{HC}$ subject.

Fig. 8. Samples of 3D PSR of $\mathrm{PRC}_{1}^{\mathrm{D} 4}$ of 4-dimensional cardiac vector: $V=\left[V_{1}, V_{2}, V_{3}, V_{4}\right]^{T}$ from $\mathrm{MI}$ subject.

Fig. 9. Samples of the Euclidian distance of 3D PSR of $\mathrm{PRC}_{1}^{\mathrm{D} 4}$ of 4-dimensional cardiac vector: $V=\left[V_{1}, V_{2}, V_{3}, V_{4}\right]^{T}$ from $\mathrm{HC}$ subject.

Fig. 10. Samples of the Euclidian distance of 3D PSR of $\mathrm{PRC}_{1}^{\mathrm{D} 4}$ of 4-dimensional cardiac vector: $V=\left[V_{1}, V_{2}, V_{3}, V_{4}\right]^{T}$ from MI subject. 


\section{Figures}

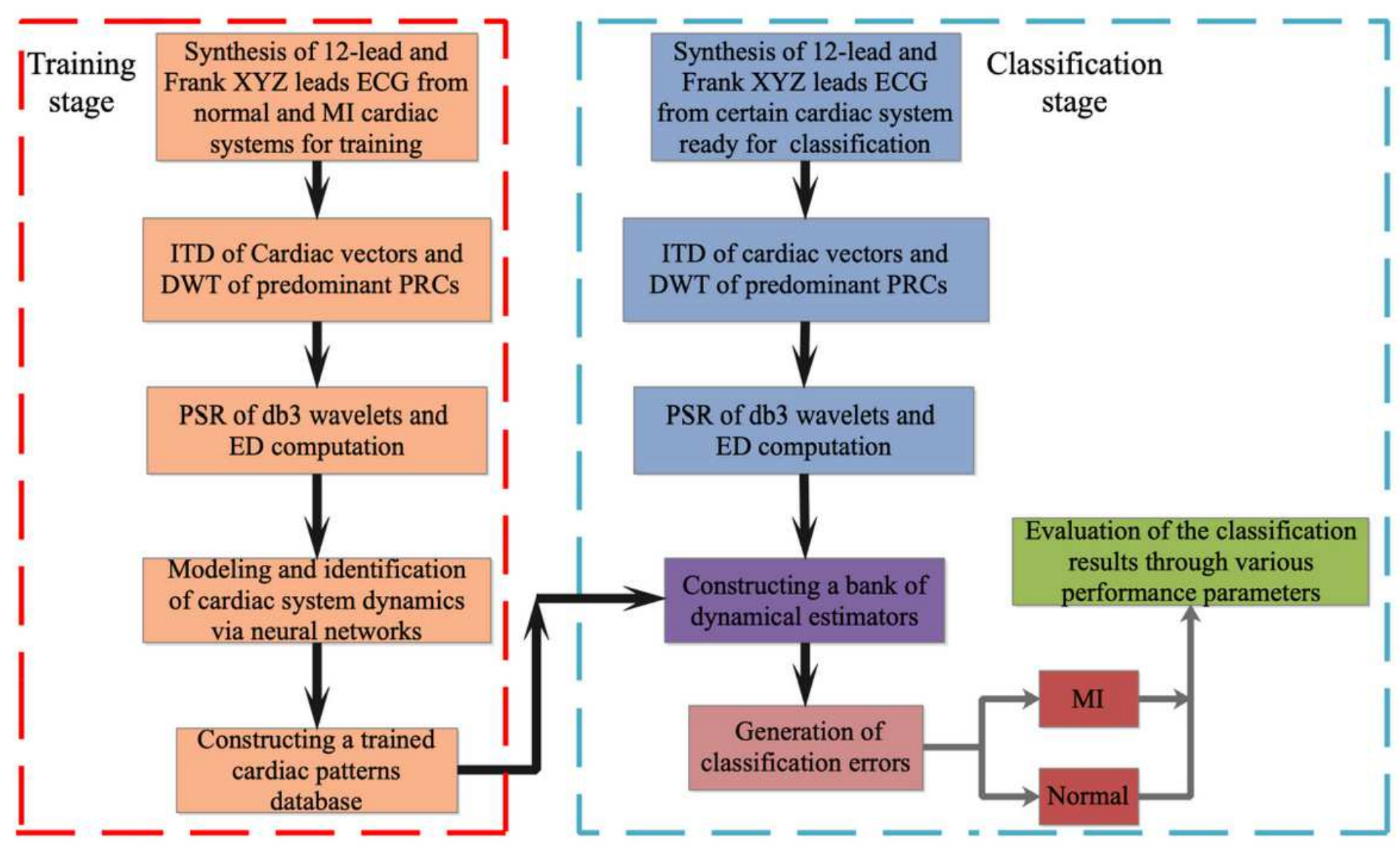

Figure 1

Flowchart of the proposed method for the MI classification using syntheses of lead system, ITD, DWT, PSR, ED and neural networks. 


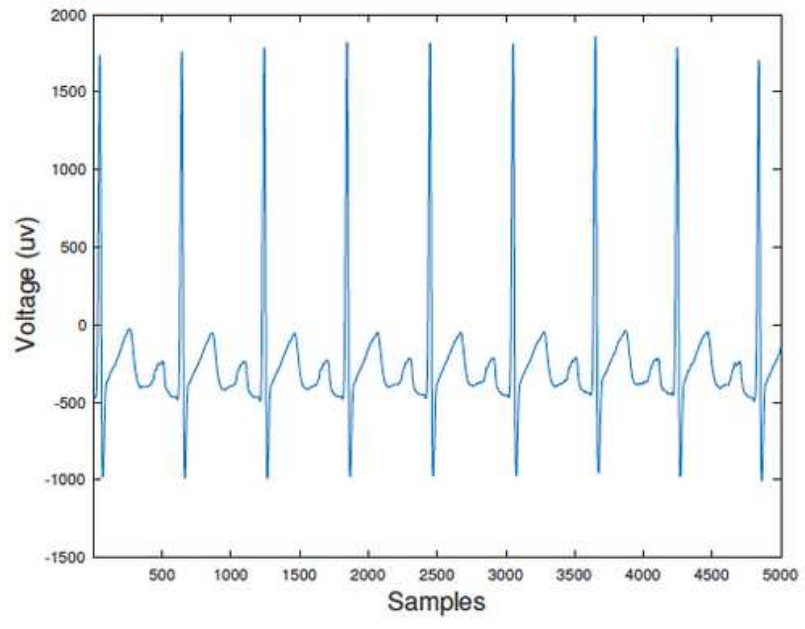

(a) $V_{1}$

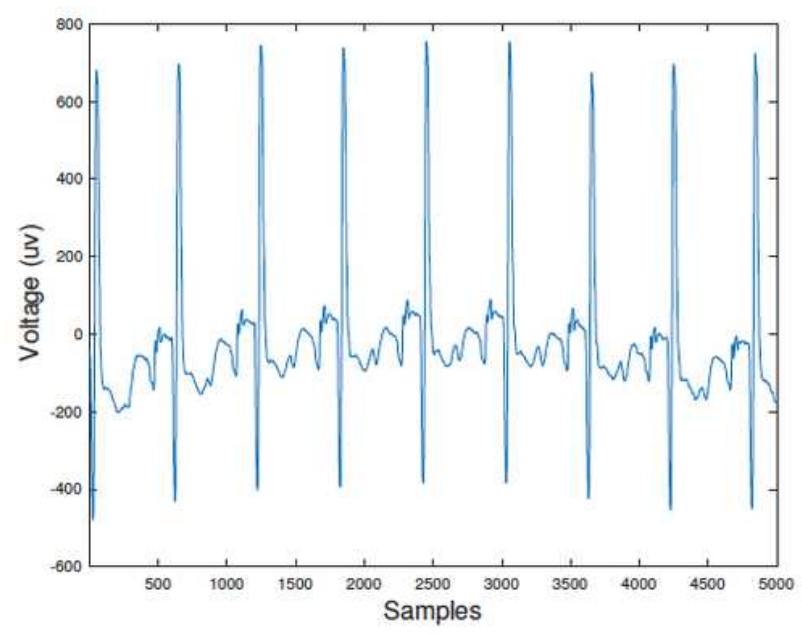

(c) $V_{3}$

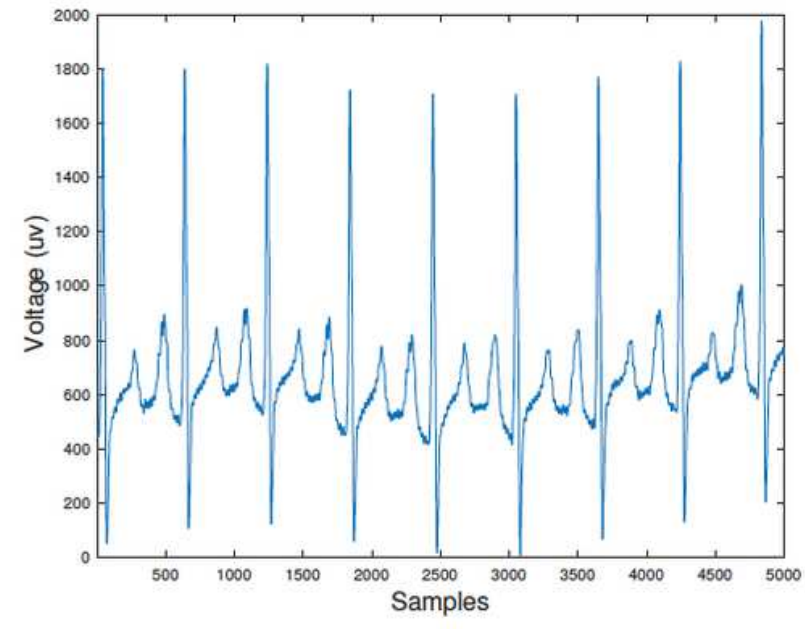

(b) $V_{2}$

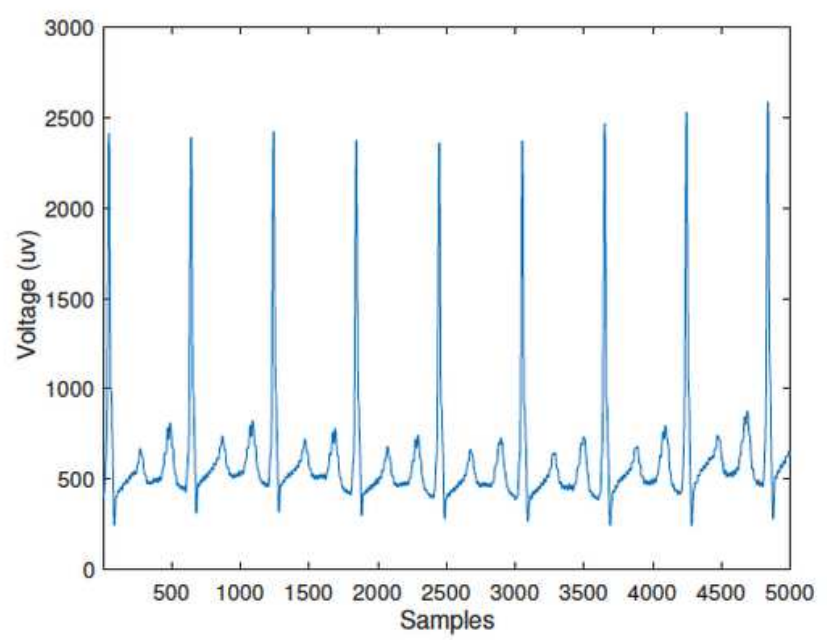

(d) $V_{4}$

Figure 2

Examples of the synthesis of 4-dimensional cardiac vector: $\mathrm{V}=[\mathrm{V} 1 ; \mathrm{V} 2 ; \mathrm{V} 3 ; \mathrm{V} 4] \mathrm{T}$ from HC subject. 


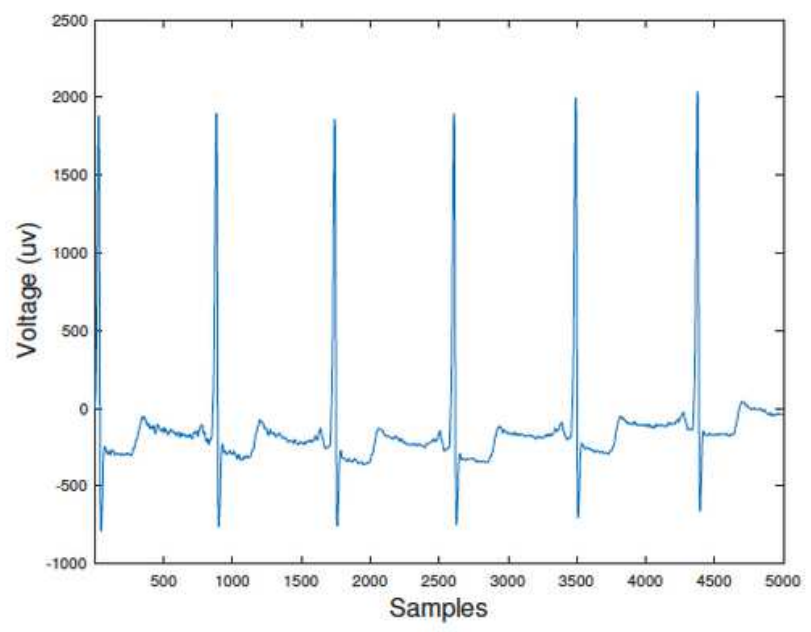

(a) $V_{1}$

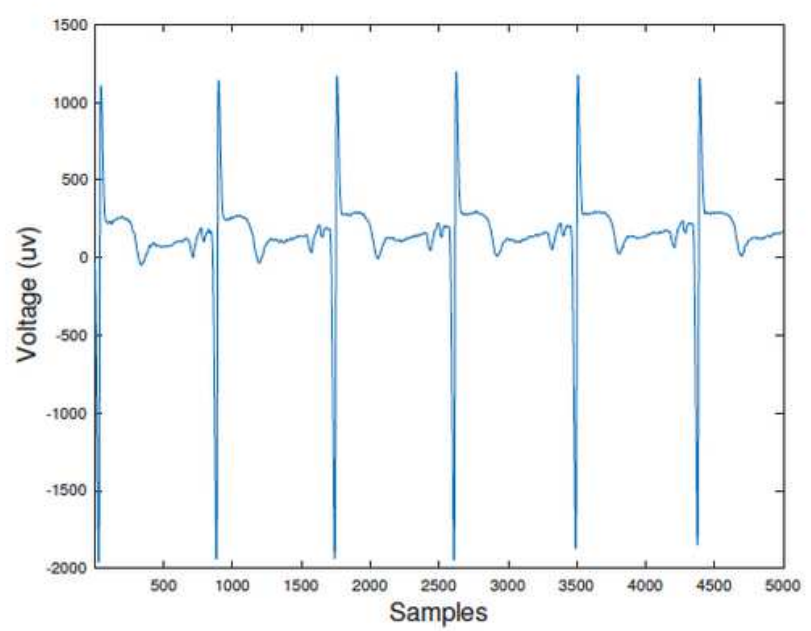

(c) $V_{3}$

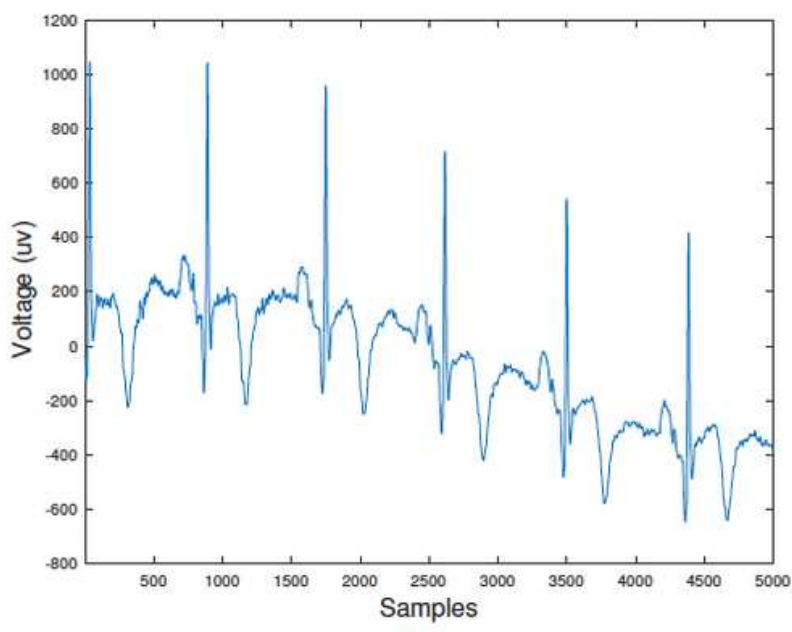

(b) $V_{2}$

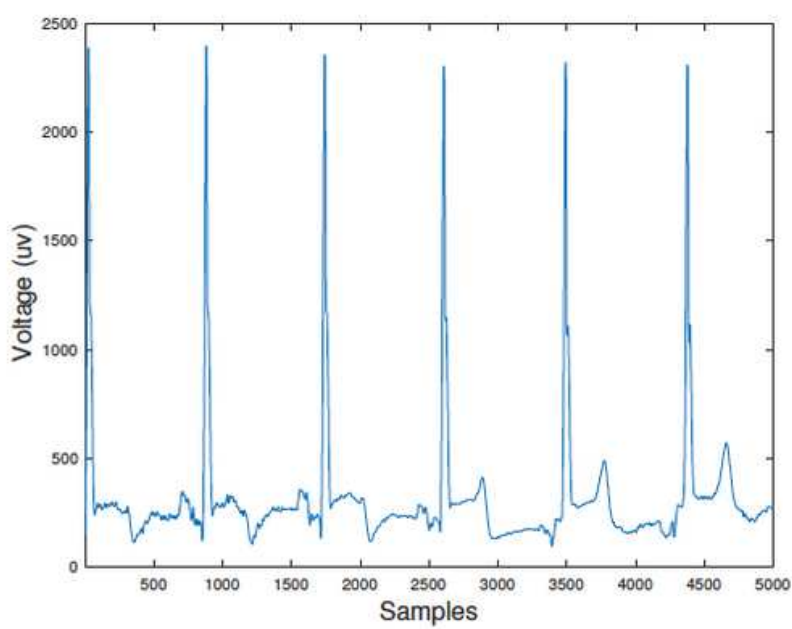

(d) $V_{4}$

\section{Figure 3}

Examples of the synthesis of 4-dimensional cardiac vector: $\mathrm{V}=[\mathrm{V} 1 ; \mathrm{V} 2 ; \mathrm{V} 3 ; \mathrm{V} 4] \mathrm{T}$ from MI subject. 


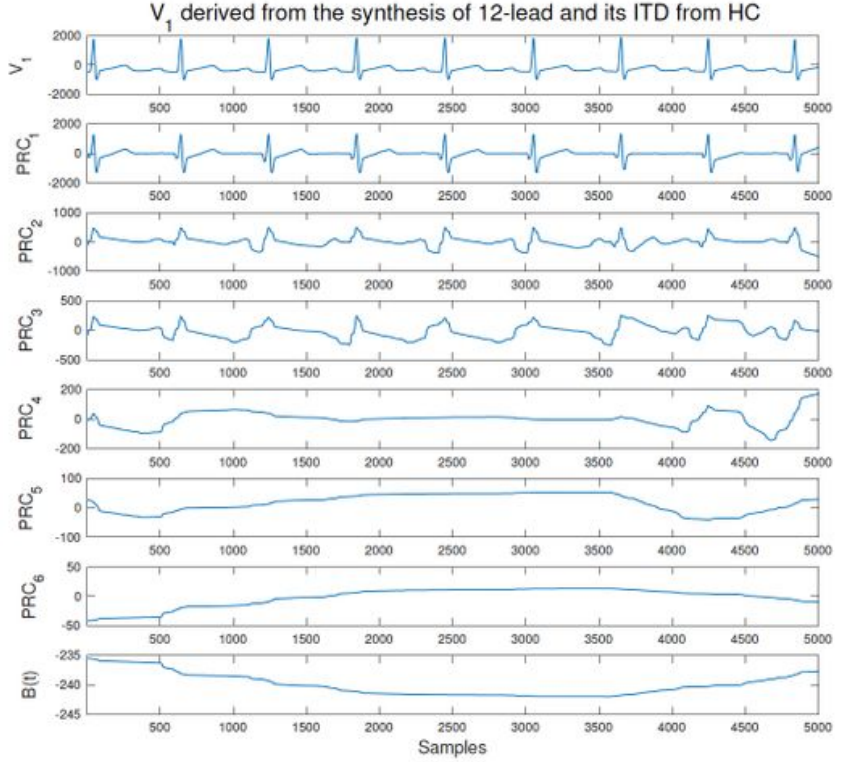

(a) Original $V_{1}$ vector and its ITD from $\mathrm{HC}$.

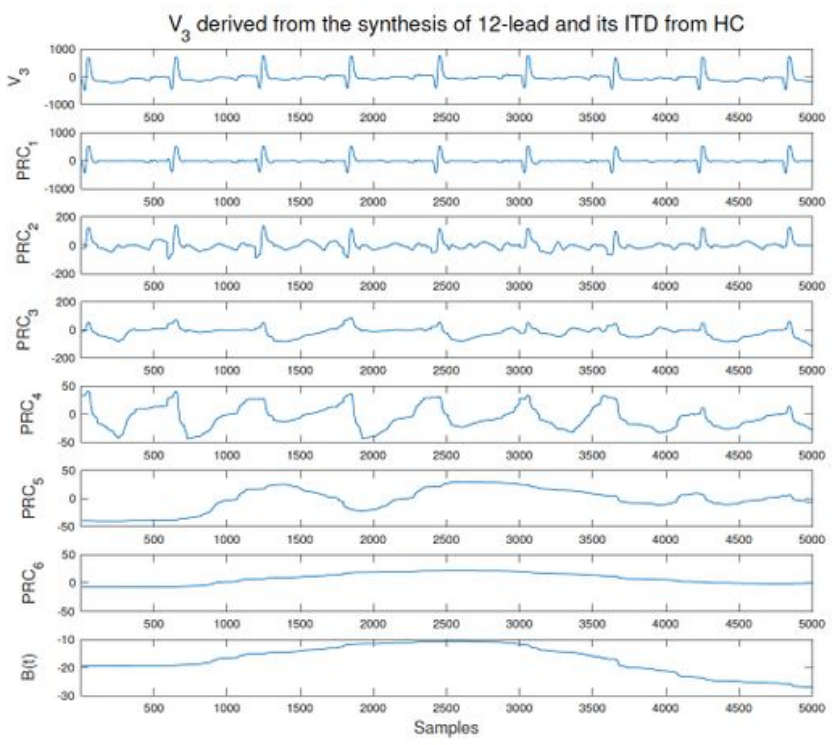

(c) Original $V_{3}$ vector and its ITD from $\mathrm{HC}$

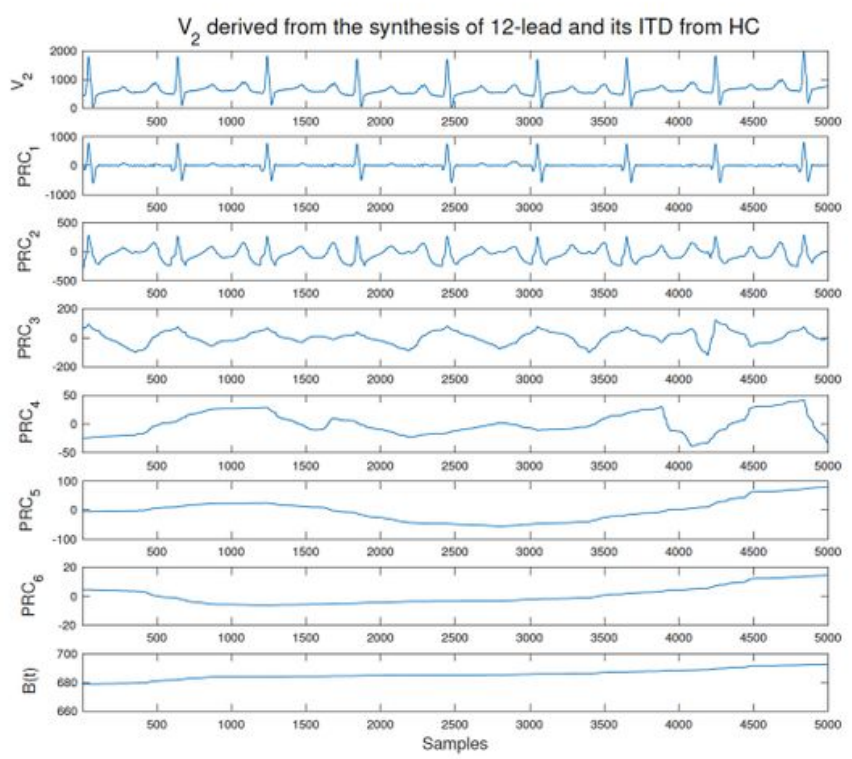

(b) Original $V_{2}$ vector and its ITD from HC

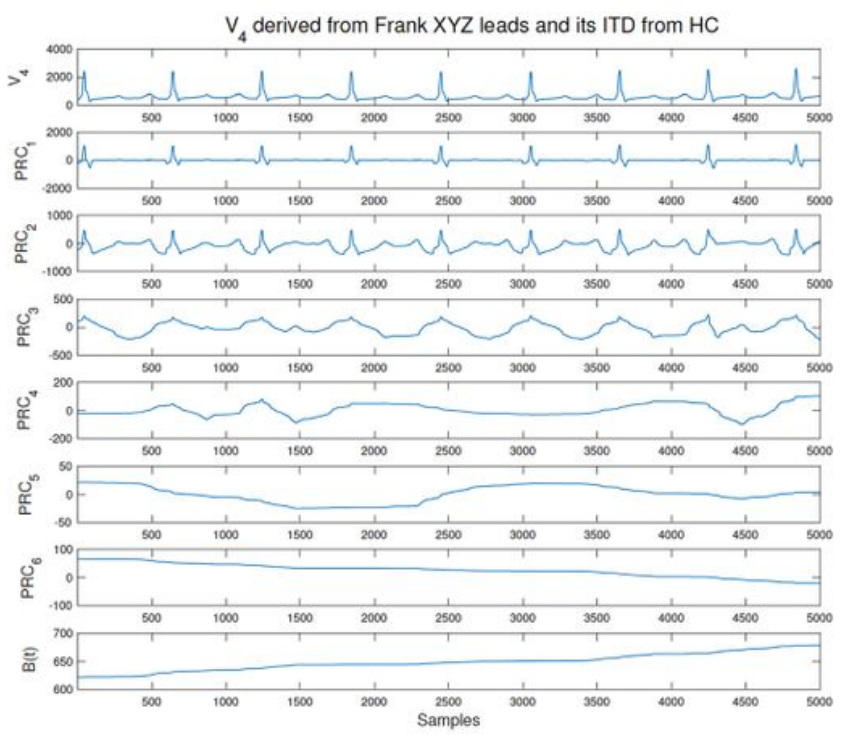

(d) Original $V_{4}$ vector and its ITD from HC

\section{Figure 4}

Samples of the ITD of 4-dimensional cardiac vector from HC subject. 


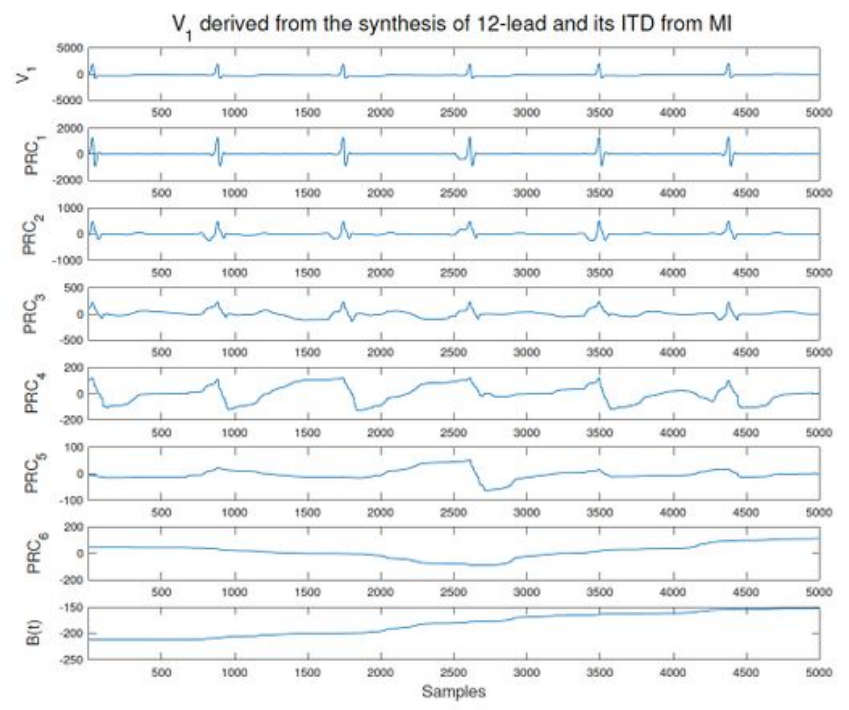

(a) Original $V_{1}$ vector and its ITD from MI.

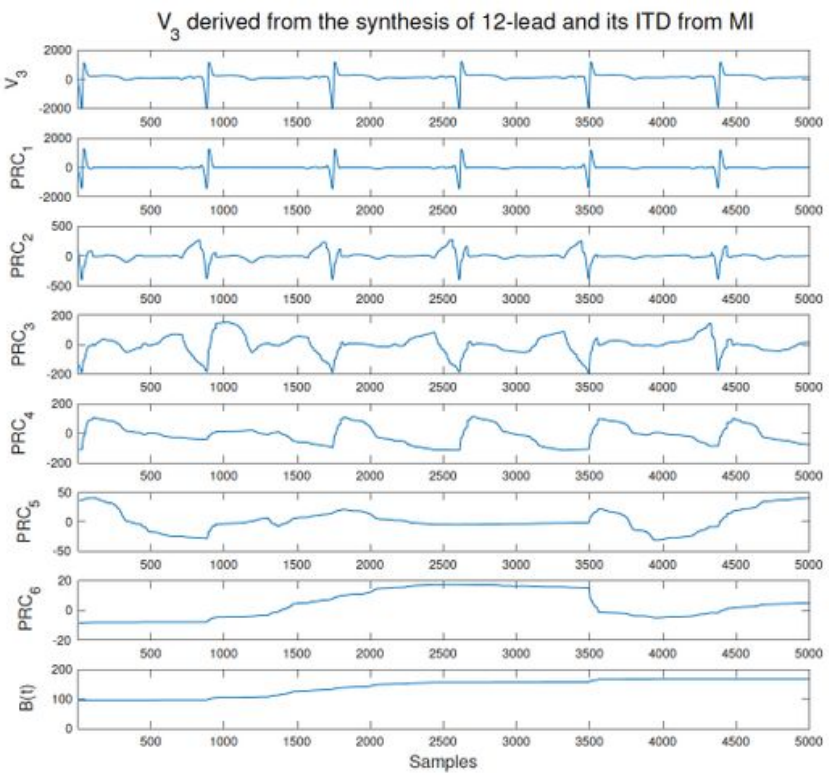

(c) Original $V_{3}$ vector and its ITD from MI

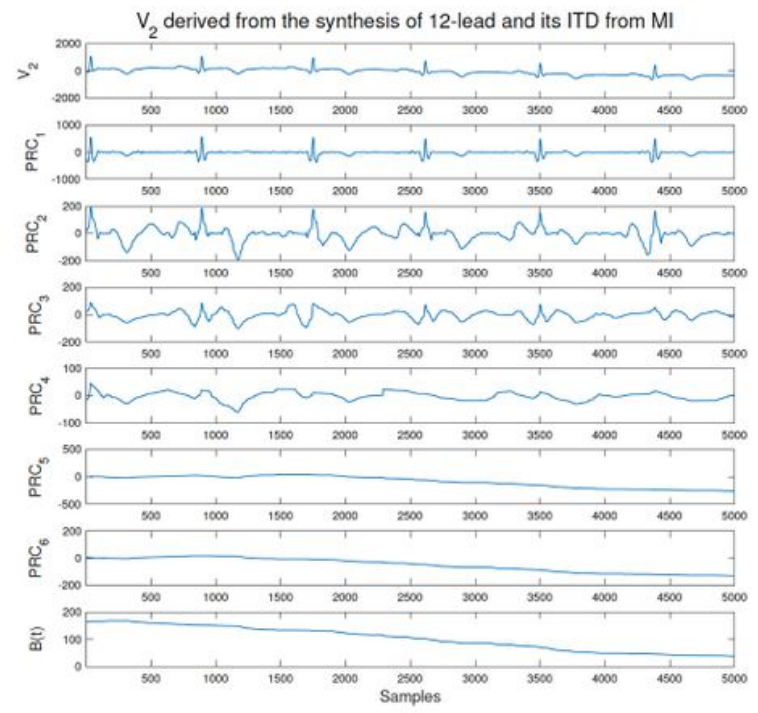

(b) Original $V_{2}$ vector and its ITD from MI

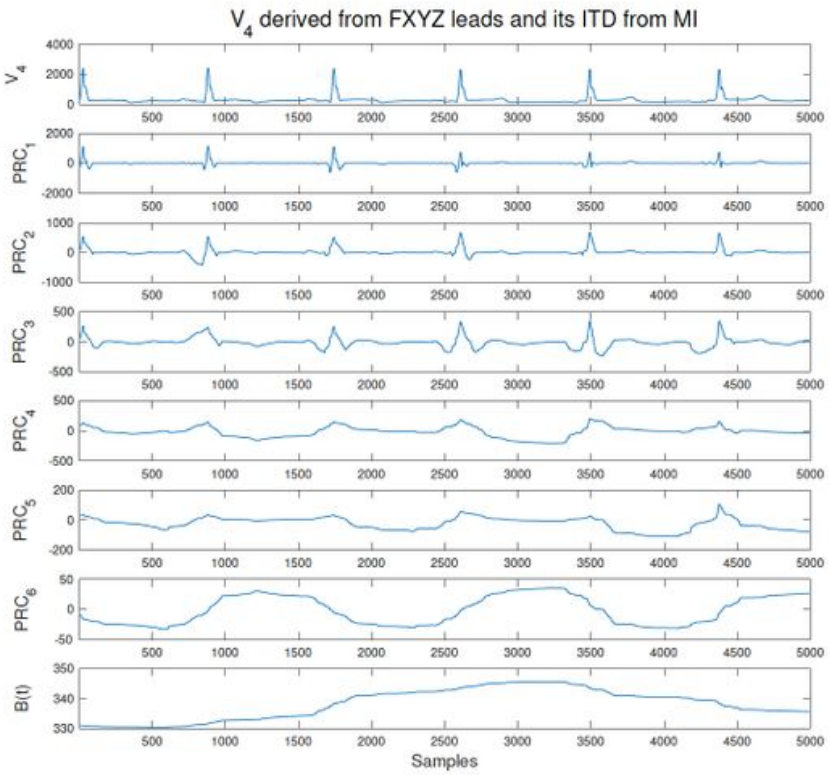

(d) Original $V_{4}$ vector and its ITD from MI

\section{Figure 5}

Samples of the ITD of 4-dimensional cardiac vector from MI subject. 

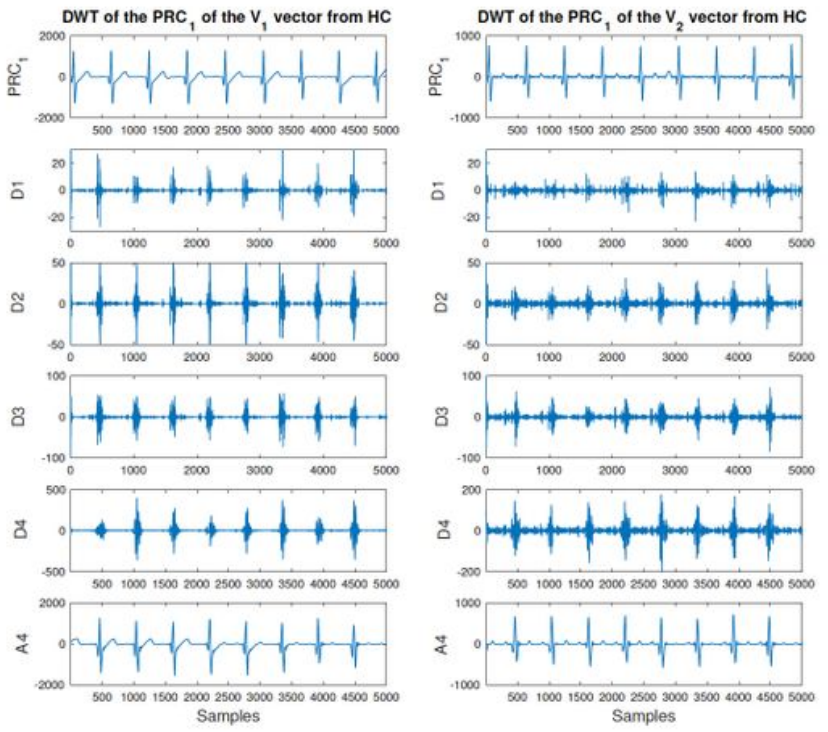

(a) Four levels DWT of $\mathrm{PRC}_{1}$ of the $V_{1}$ and $V_{2}$ vectors from HC.
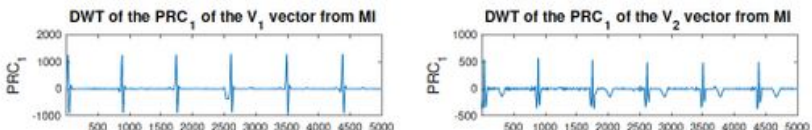

$=\underbrace{20}_{2000}$
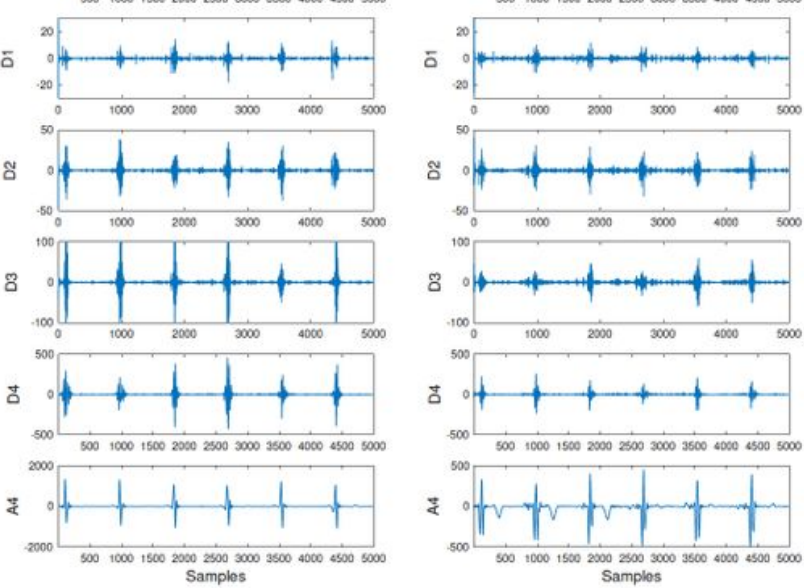

(c) Four levels DWT of $\mathrm{PRC}_{1}$ of the $V_{1}$ and $V_{2}$ vectors from MI

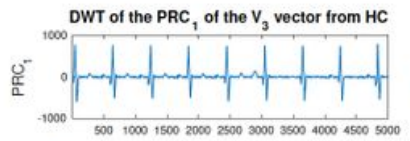

DWT of the PRC, of the $\mathrm{V}_{4}$ vector from HC
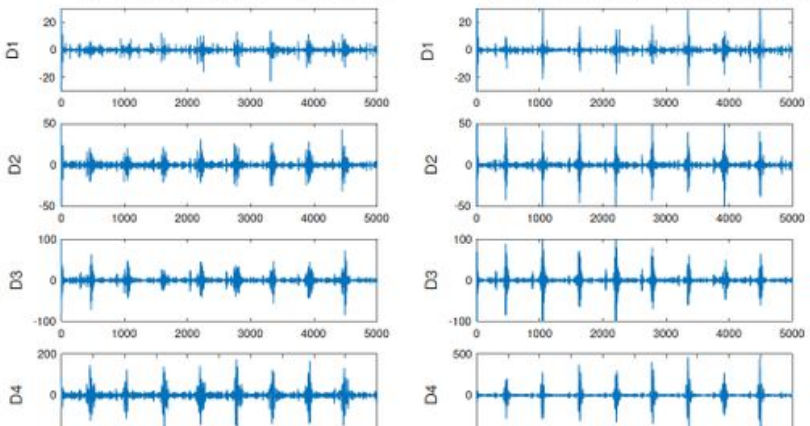

${ }_{200} 5000100015002000250035000350004000455005000$

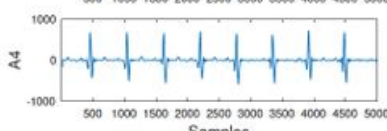

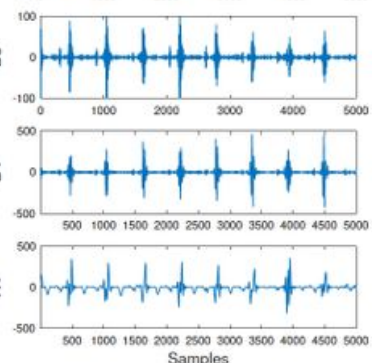

Samples

(b) Four levels DWT of $\mathrm{PRC}_{1}$ of the $V_{3}$ and $V_{4}$ vectors from $\mathrm{HC}$.
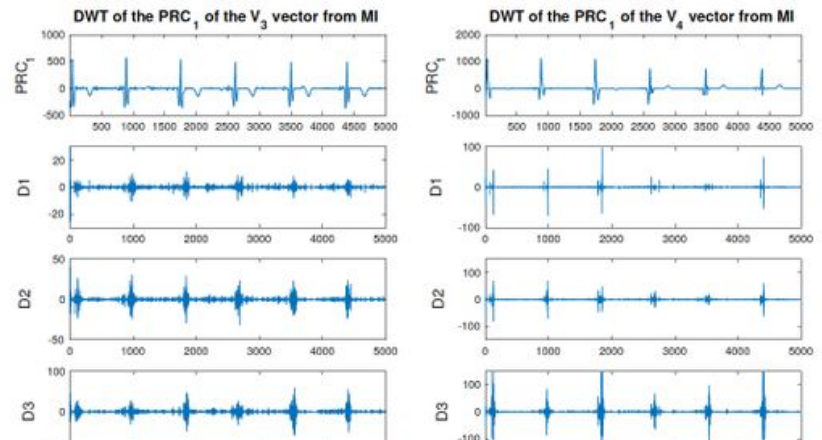

${ }_{1000} \underbrace{}_{2000}{ }_{2000}$

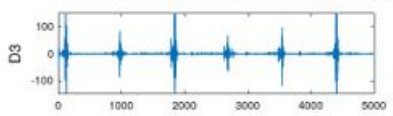

¿ of +++++

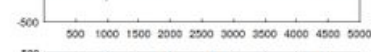
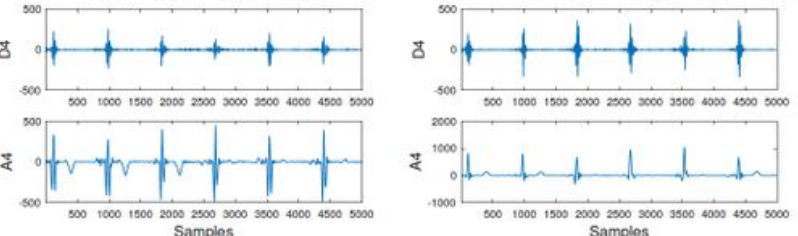

(d) Four levels DWT of $\mathrm{PRC}_{1}$ of the $V_{3}$ and $V_{4}$ vectors from MI.

\section{Figure 6}

Samples of four levels DWT of PRC1 of the 4-dimensional cardiac vector: V = [V1; V2; V3; V4]T from HC and Ml subjects. 


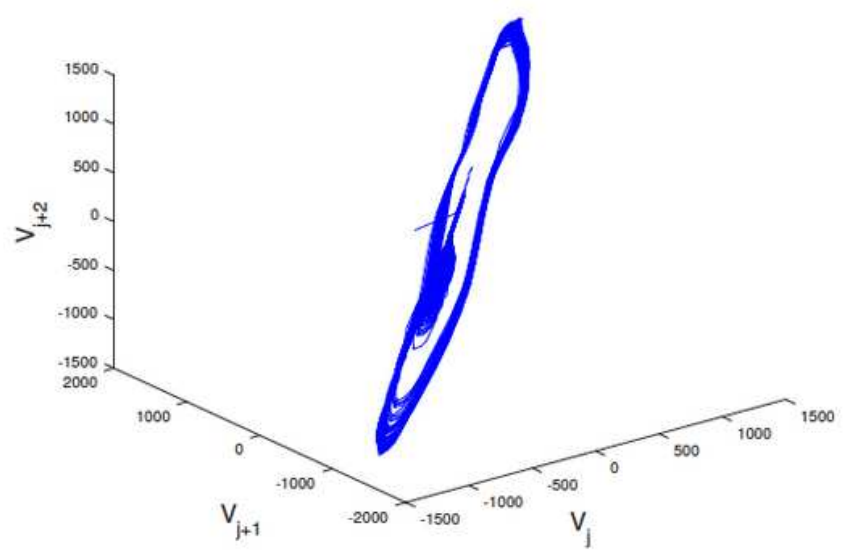

(a) 3D PSR of $\mathrm{PRC}_{1}^{\mathrm{D} 4}$ for $V_{1}$.

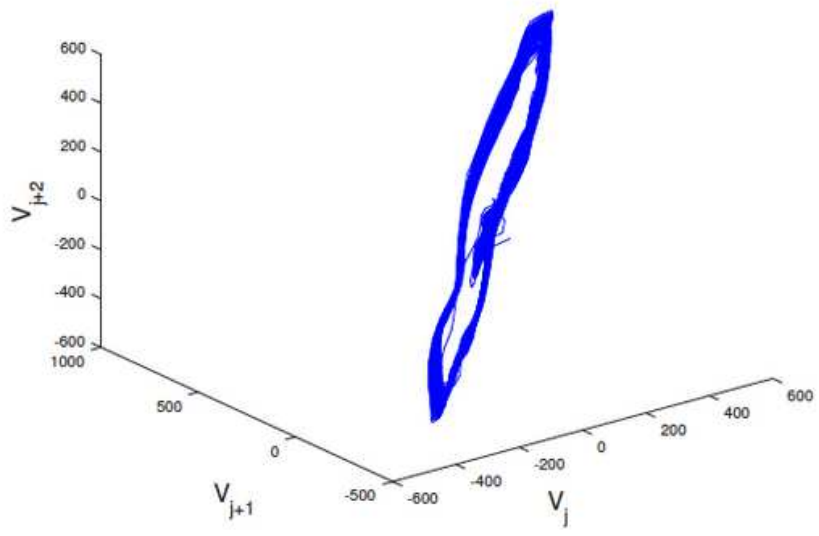

(c) 3D PSR of $\mathrm{PRC}_{1}^{\mathrm{D} 4}$ for $V_{3}$.

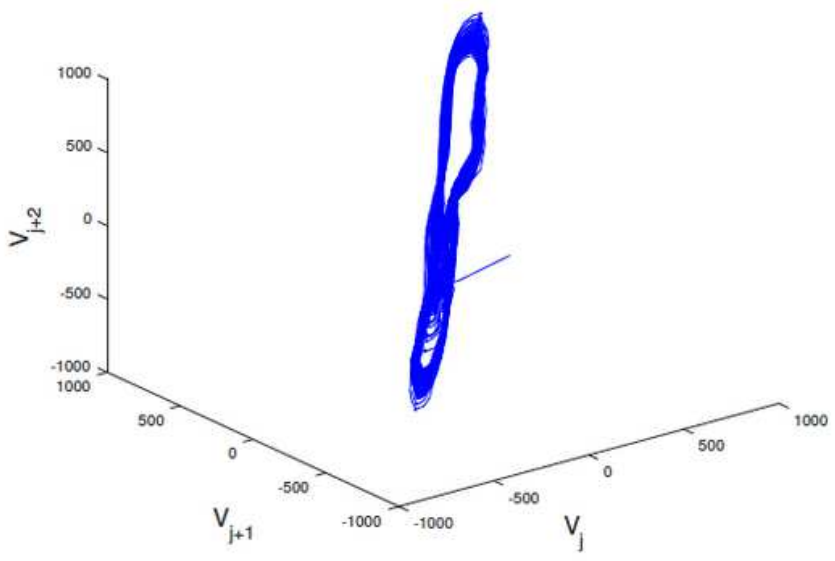

(b) 3D PSR of $\mathrm{PRC}_{1}^{\mathrm{D} 4}$ for $V_{2}$.

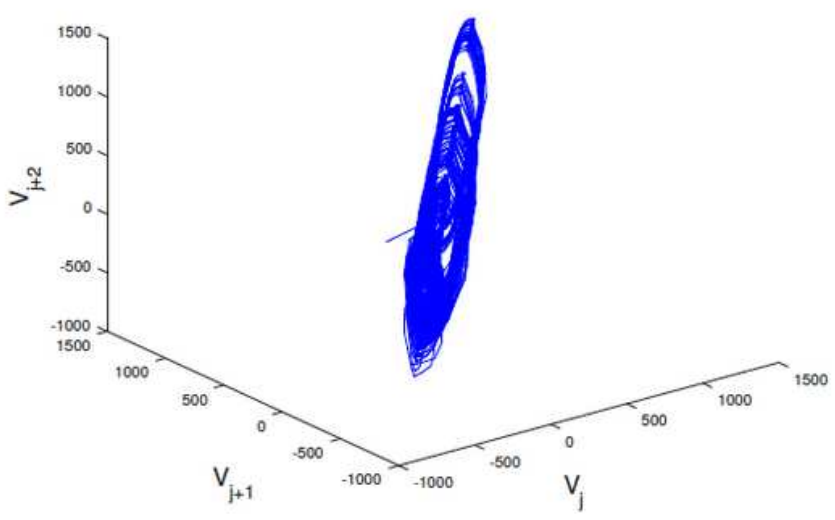

(d) 3D PSR of $\mathrm{PRC}_{1}^{\mathrm{D} 4}$ for $V_{4}$.

\section{Figure 7}

Samples of 3D PSR of PRCD4 1 of 4-dimensional cardiac vector: V = [V1; V2; V3; V4]T from HC subject. 


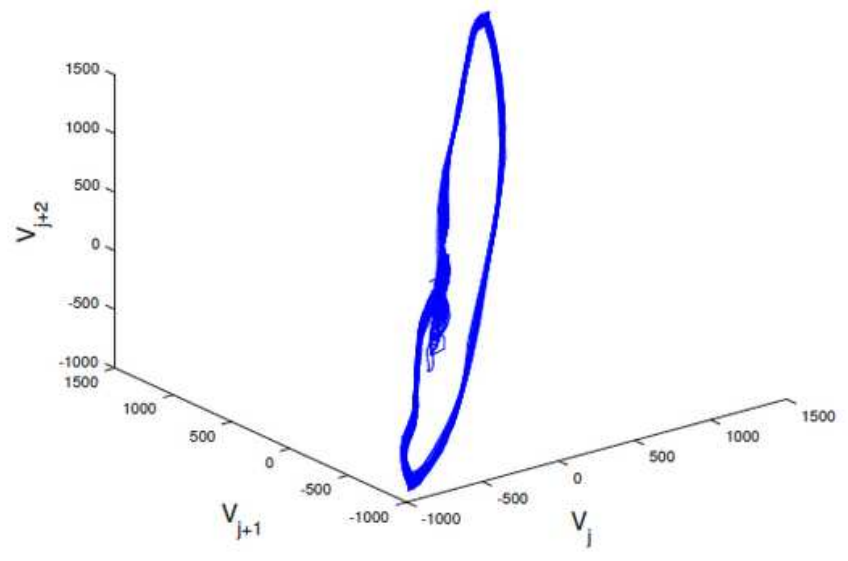

(a) 3D PSR of $\mathrm{PRC}_{1}^{\mathrm{D} 4}$ for $V_{1}$.

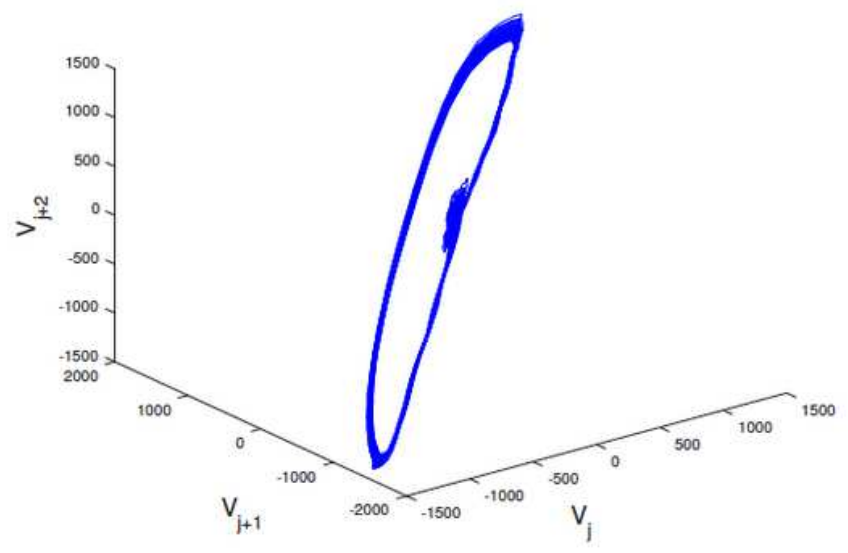

(c) 3D PSR of PRC 14 for $V_{3}$.

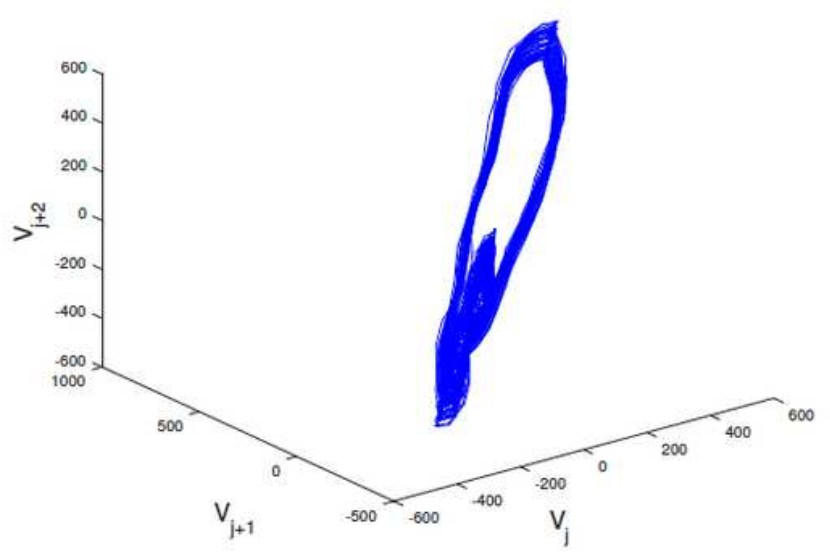

(b) 3D PSR of $\mathrm{PRC}_{1}^{\mathrm{D} 4}$ for $V_{2}$.

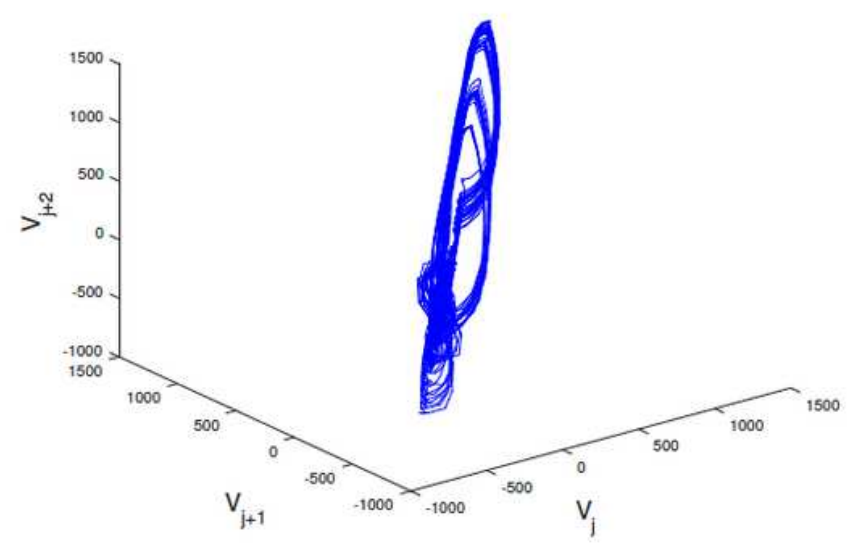

(d) 3D PSR of $\mathrm{PRC}_{1}^{\mathrm{D} 4}$ for $V_{4}$.

Figure 8

Samples of 3D PSR of PRCD4 1 of 4-dimensional cardiac vector: V = [V1; V2; V3; V4]T from MI subject. 


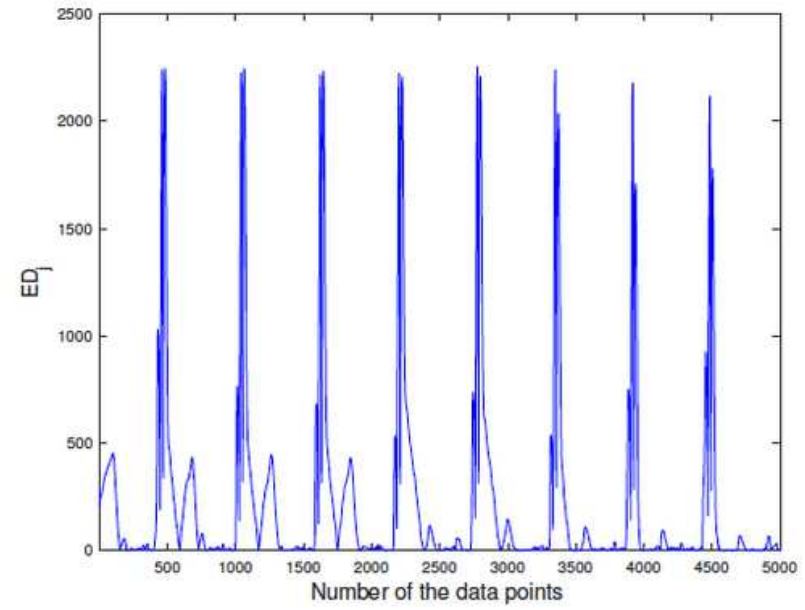

(a) Euclidian distance of 3D PSR of $\mathrm{PRC}_{1}^{\mathrm{D} 4}$ for $V_{1}$.

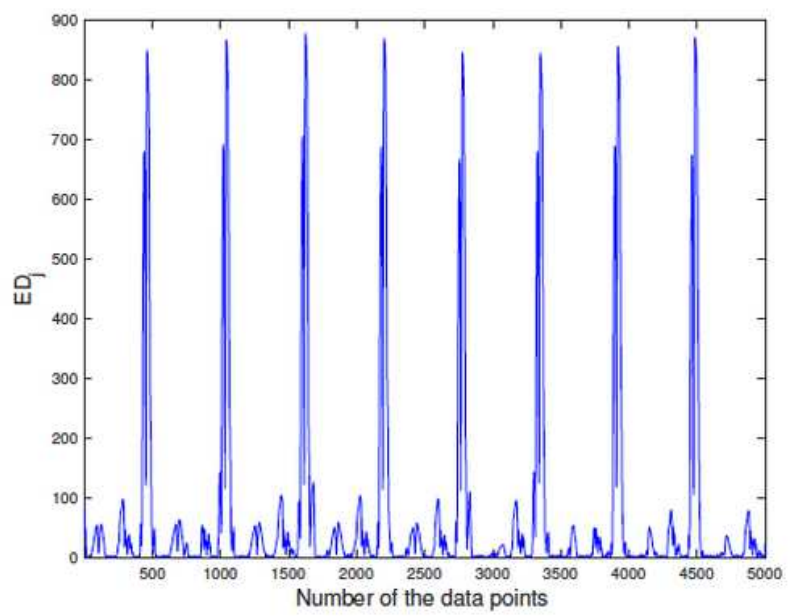

(c) Euclidian distance of 3D PSR of $\mathrm{PRC}_{1}^{\mathrm{D} 4}$ for $V_{3}$.

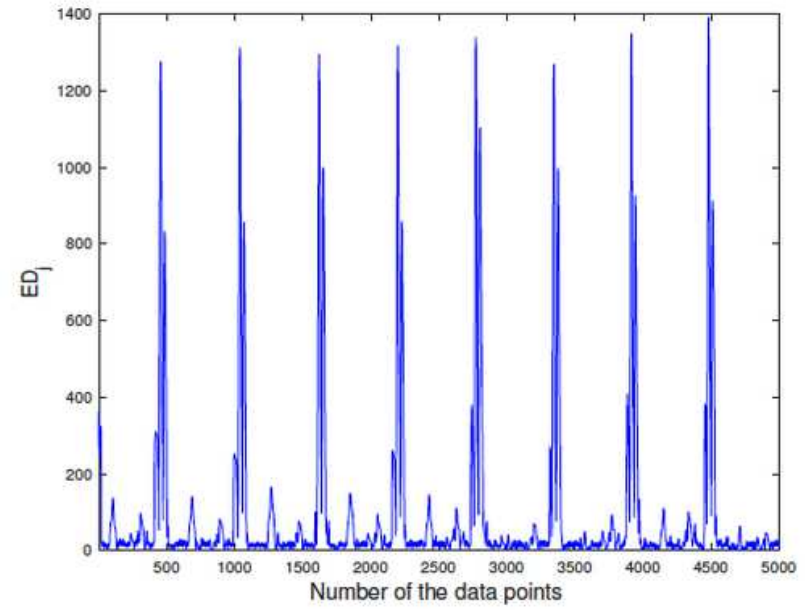

(b) Euclidian distance of 3D PSR of $\mathrm{PRC}_{1}^{\mathrm{D} 4}$ for $V_{2}$.

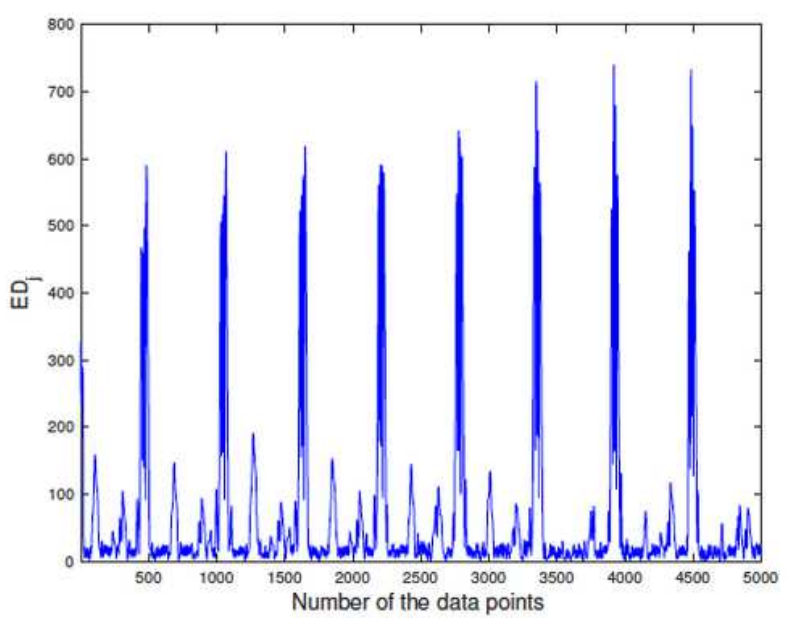

(d) Euclidian distance of 3D PSR of $\mathrm{PRC}_{1}^{\mathrm{D} 4}$ for $V_{4}$.

\section{Figure 9}

Samples of the Euclidian distance of 3D PSR of PRCD4 1 of 4-dimensional cardiac vector: V = [V1; V2; V3; V4]T from HC subject. 


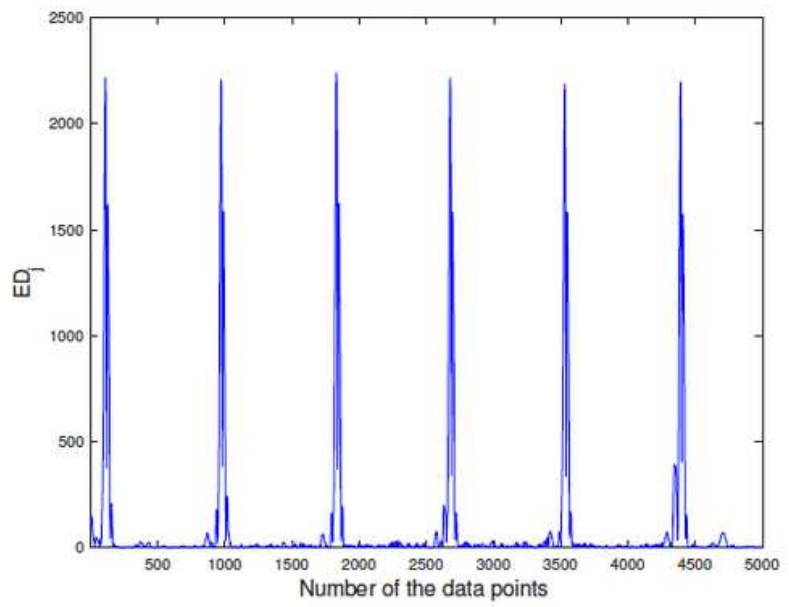

(a) Euclidian distance of 3D PSR of $\mathrm{PRC}_{1}^{\mathrm{D} 4}$ for $V_{1}$.

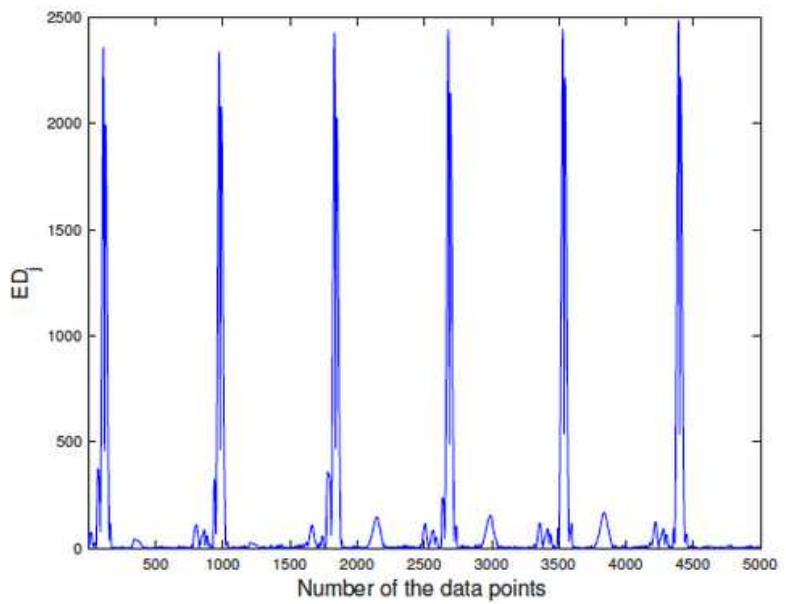

(c) Euclidian distance of 3D PSR of $\mathrm{PRC}_{1}^{\mathrm{D} 4}$ for $V_{3}$.

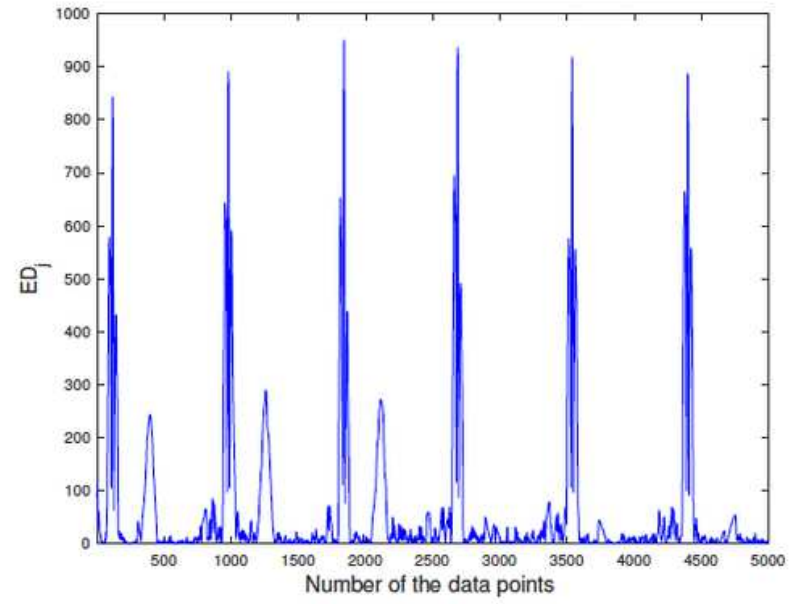

(b) Euclidian distance of 3D PSR of $\mathrm{PRC}_{1}^{\mathrm{D} 4}$ for $V_{2}$.

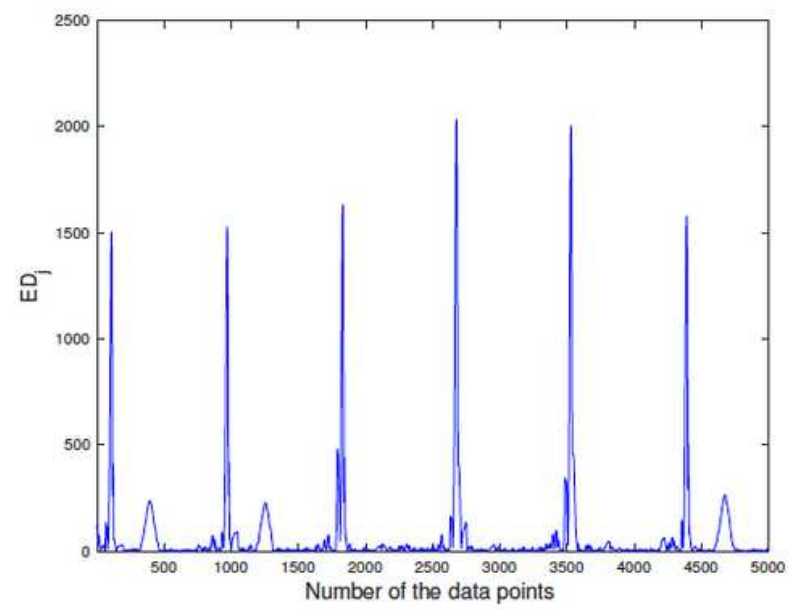

(d) Euclidian distance of 3D PSR of $\mathrm{PRC}_{1}^{\mathrm{D} 4}$ for $V_{4}$.

Figure 10

Samples of the Euclidian distance of 3D PSR of PRCD4 1 of 4-dimensional cardiac vector: V = [V1; V2; V3; V4]T from MI subject. 